\title{
Fauna and distribution of spiders (Aranei) of the arctic tundra in northern Yamal Peninsula, Russia
}

\author{
Фауна и зонально-ландшафтное распределение пауков (Aranei) \\ в арктических тундрах северного Ямала, Россия
}

\author{
Andrei V. Tanasevitch, Leonid B. Rybalov \\ А.В. Танасевич, А.Б. Рыбалов \\ Institute of Ecology and Evolution, Russian Academy of Sciences, Leninsky Prospect 33, Moscow 119071, Russia. E-mail: \\ tanasevitch@gmail.com; 1rybalov52@mail.ru \\ Институт проблем экологии и эволюции РАН, Ленинский проспект 33, Москва 119071, Россия.
}

KEY WORDS: Spiders, Arctic, arctic tundra, chorology, Yamal Peninsula.

КЛЮЧЕВЫЕ СЛОВА: Пауки, Арктика, арктическая тундра, хорология, п-ов Ямал.

ABSTRACT. Thirty five spider species are recorded from the arctic tundra of northern Yamal Peninsula, of which eight are new to the Yamal fauna: Arcterigone pilifrons (L. Koch, 1879), Hilaira nivalis Holm, 1937, Mecynargus tundricola Eskov, 1988, Mughiphantes sobrius (Thorell, 1872), Paraglyphesis polaris Eskov, 1991, Perro polaris (Eskov, 1986), Semljicola simplex (Kulczyński, 1908) and Silometopoides pampia (Chamberlin, 1948). Of them, the Yamal records of the three latest species represent the westernmost limit of their known distribution. In terms of regional composition, the spider fauna of northern Yamal is indeed Siberian, which sets it apart from the fauna of southern Yamal containing significantly more European elements. In zonal terms, the araneofauna of northern Yamal has an arctic appearance and contains only two polyzonal elements, with the rest of species displaying arctic, arcto-alpine or arcto-boreal distributional patterns. Despite the most pessimal environmental conditions, the zonal tundra communities yield a comparable number of species to that of intrazonal biotopes which are characterized by a significantly smoother gradient of climatic factors. Most species of local, arctic tundra populations are polytopic or eurytopic, i.e. the species inhabiting the entire landscape profile and all/most of the vegetation types, showing no preference to a specific landscape element or a plant community. Only two species could be characterized as typical zonal elements: arctic Halorates spetsbergensis (Thorell, 1872) and Xysticus albidus Grese, 1909; and five species as typical intrazonal elements: Bathyphantes humilis (L. Koch, 1879), Hilaira incondita (L. Koch, 1879), H. proletaria (L. Koch, 1879), Pelecopsis parallela (Wider, 1834) and Silometopoides pampia (Chamberlin, 1948).

РЕЗЮМЕ. В арктических тундрах северного Ямала обнаружено 35 видов пауков, 8 из которых впервые отмечены в фауне п-ова: Arcterigone pilifrons (L.
Koch, 1879), Hilaira nivalis Holm, 1937, Mecynargus tundricola Eskov, 1988, Mughiphantes sobrius (Thorell, 1872), Paraglyphesis polaris Eskov, 1991, Perro polaris (Eskov, 1986), Semljicola simplex (Kulczyński, 1908) и Silometopoides pampia (Chamberlin, 1948). Для трёх последних видов Ямал — крайняя западная область их распространения. В региональном плане, фауна пауков северного Ямала типично сибирская, что отличает ее от фауны южного Ямала, где европейские элементы представлены существенно богаче. В зональном аспекте аранеофауна северного Ямала имеет арктический облик и содержит лишь два полизональных вида, остальные имеют арктический, аркто-альпийский или аркто-бореальный тип распространения. Зональные тундровые сообщества, несмотря на наиболее пессимальные условия среды, по числу обитающих здесь видов не уступают интразональным биотопам, где градиент климатических факторов существенно сглажен. Большинство видов местного населения арктических тундр являются политопными и эвритопными, т.е. видами, осваивающими весь ландшафтный профиль и типы растительности, не демонстрируя явной приуроченности к какому-либо определенному элементу ландшафта или растительной ассоциации. Лишь два вида могут быть охарактеризованы как типично зональные: арктические Halorates spetsbergensis (Thorell, 1872) and Xysticus albidus Grese, 1909, и пять видов, как типично интразональные: Bathyphantes humilis (L. Koch, 1879), Hilaira incondita (L. Koch, 1879), H. proletaria (L. Koch, 1879), Pelecopsis parallela (Wider, 1834) и Silometopoides pampia (Chamberlin, 1948).

\section{Introduction}

Yamal Peninsula is situated in the extreme northwestern part of Siberia and belongs to Yamal-Nenets Autonomous Region of Tyumen Area, Russia (see Map). 
In the present paper, northern Yamal is accepted as the territory lying north of $70.5^{\circ} \mathrm{N}$, i.e. the part of the Peninsula that, according to Rebristaya [2013], lies completely in the arctic tundra subzone of the tundra belt. Araneologically, this part of the Peninsula remains practically unexplored. There are only two species listed from the western coast: viz., Halorates spetsbergensis (Thorell, 1872) and Erigone arctica sibirica Kulczyñski, 1908 [Holm, 1973] (see Map). The identification of the latter species is obviously mistaken and should actually be referred to $E$. a. palaearctica Brændegaard, 1934 (see below). The territory of central Yamal lying in the hypoarctic tundra subbelt [sensu Rebristaya, 2013] is studied much better, with 28 spider species being reported from there to date [Tanasevitch et al., 2009]. The entire araneofauna of Yamal consists of about 180 species, of which the majority are known from the southern part of the Peninsula, or to be exact, from the basin of Shchyuchya and Khadyta rivers situated at the base of the Peninsula [Esyunin, Efimik, 1996; Tanasevitch et al., 2009] (see Map).

The present paper is devoted to the fauna and chorology of spiders occurring in the arctic subzone of the tundra belt of northern Yamal.

\section{Material and methods}

The present study is based on extensive spider material collected by Leonid Rybalov, Andrei Tanasevitch and Alexander Bastrakov from 31 localities (61 sites) of the northern part of Yamal Peninsula in 2013-2014. Small additional material was available from Anatoly Babenko, Marina Khrisanova and Vladimir Onufriev. All specimens are deposited in the Zoological Museum of the Moscow State University, Moscow, Russia (curator - K.G. Mikhailov).

Spiders were collected by sifting moss and litter, soil sampling, pitfall trapping and hand collecting. In total, over 1400 adult spider specimens were collected and examined.

The scheme proposed by Yu.I. Chernov [1978] for classifying plant communities of the Arctic has been used for an analysis of zonal-landscape distribution of tundra spiders. Based on this classification, there are three main zonal types of plant communities in the tundra (for more details, see Tanasevitch \& Koponen [2007]):

Zonal communities - the plant communities that are restricted to the watershed of one zone, defining the vegetation zonal face of the landscape. In zonal communities, abiotic factors are more extreme compared to those in intrazonal communities and therefore living conditions are more adverse. In northern Yamal Peninsula, the zonal plant associations are represented by three main types of tundra vegetation: moss-, lichenmoss-, sedge-lichen-moss-communities, with rare and insignificant proportion of undershrubs, often with patches of bare ground. Shrubs are practically absent, except for singular and undersized Betula nana or willows (Salix polaris, S. glauca). Of the undershrubs, the main composing elements are Cassiopa tetragona, Vac- cinium vitis-idaea è Rubus chamaemorus. Dwarf shrubs Arctous alpina and Empetrum hermaphroditum, which are common in the hypoarctic tundra subzone, are rare and scanty. The dominants among mosses are Dicranum congestum, Dicranum flexicaule, Hylocomium splendens, Mnium sp., Pleurozium schreberi; the dominating lichens are Cetraria islandica, C. nigricans, Cladonia arbuscula, $C$. rangiferina and Alectoria nigricans. Sedges (Carex arctisibirica) and grasses (Arctagrostis latifolia, Poa arctica, P. alpigena, Deschampsia borealis) are rare and scanty.

Intrazonal communities - the plant communities that are distributed across one/several zones; the typical examples are swamps and river valley vegetation: e.g., willow stands on floodplains, meadows, pebble banks, rocky and/or grassy slopes, forest stands on river banks or river terraces, etc. Commonly, parts of intrazonal communities are situated on the watershed among zonal vegetation types: these are willow stands in depressions, shrubby lake shores, flat-hill peatbogs with sedge fens, or sphagnum bogs in depressions, grassy slopes of small brooks, etc. The intrazonal communities are characterized by a smoother gradient of climatic factors (especially, of temperature and humidity), making living conditions there significantly better (less pessimal). In northern Yamal, the intrazonal communities are represented by numerous variants of swampy tundras, small meadows, diverse herbs-willow communities on river terraces and ribbon-shaped associations in depressions among the polygonal tundra. The most typical intrazonal communities are as follows:

1. Microdepressions on watershed:

- moss-grass-sedge ribbon-shaped associations in depressions among the polygonal tundra with Pleurozium schreberi, Hylocomium splendens, Arctagrostis latifolia, Carex arctisibirica, Poa alpina, etc.

\section{Swamps:}

- Sphagnum swamps with Sphagnum warnstorfii, S. squarrosum, S. cuspidatum, Carex aquatilis, Comarum palustre, etc.;

- Eriophorum-sedge-moss swamps with Salix glauca, Carex aquatilis, Carex rariflora, Comarum palustre, Eriophorum media, Aulacomnium turgidum, Calliergon richardsonii, Sphagnum cuspidatum, S. warnstorfii, etc.

3. Floodplains:

- Salix glauca \& S. reticulata stands with diverse herbs-sedge associations on high floodplains with $A s-$ tragalus subpolaris, Carex arctisibirica, Festuca cryophila, Peltigera sp., etc.;

- swampy diverse herbs-sedge regularly inundated meadows near lakes with Arctagrostis latifolia, Carex aquatilis, C. arctisibirica, Eriophorum polystachion, E. medium, Comarum palustre, Ranunculus pallasii, Aulacomnium palustre, A. turgidum, Calliergon richardsonii, Sphagnum squarrosum, etc.;

- wet sedge-moss communities among streams, in depressions with Carex aquatilis, C. arctisibirica, Calliergon cavolifolia, C. richardsonii, Aulacomnium palustre, etc.; 


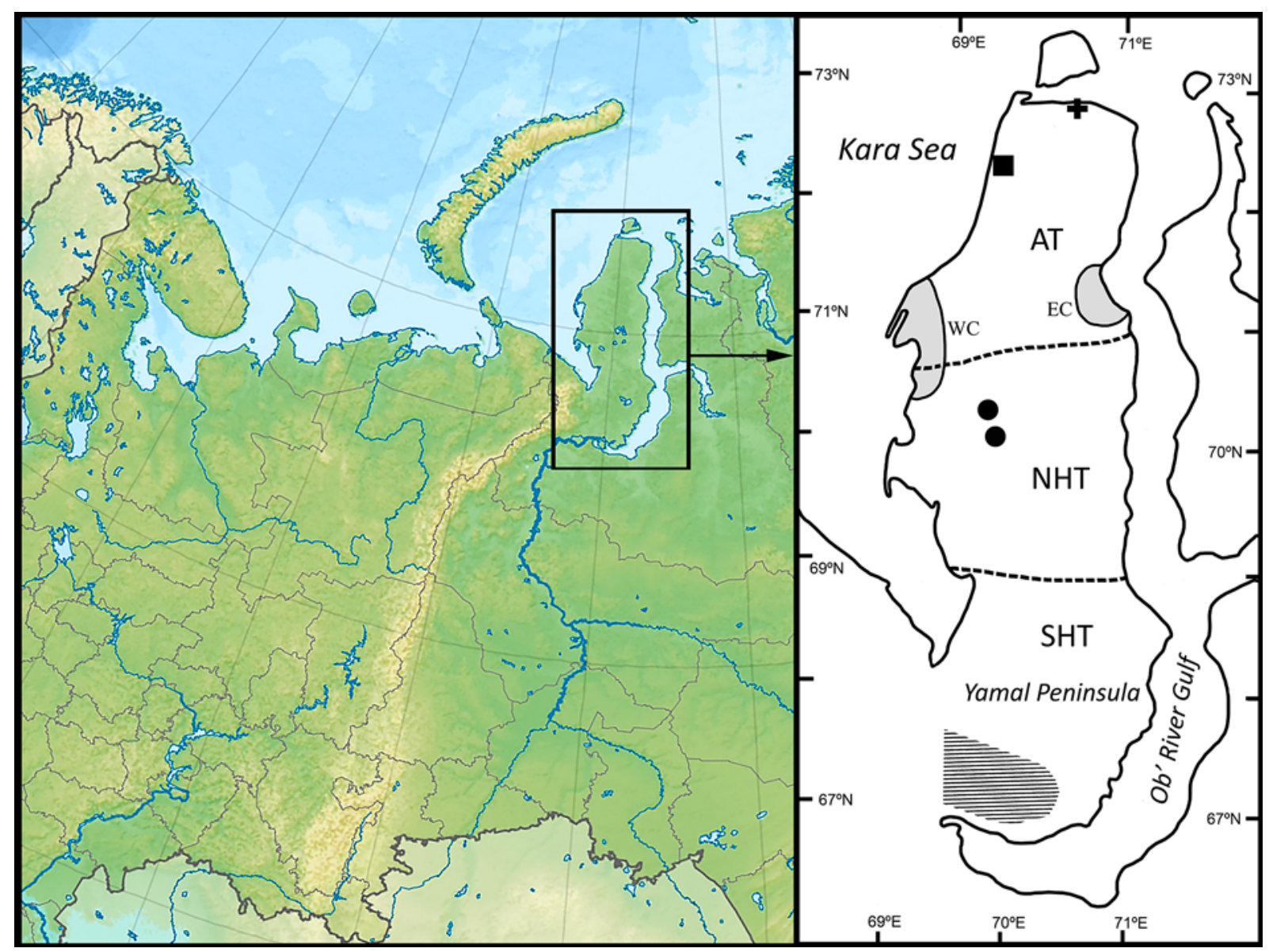

Map. Collecting areas and localities in Yamal Peninsula. Filled areas — regions of the present study in 2013-2014: WC — western coast region; EC - eastern coast region. Cross - A. Babenko in 1994; square - Holm [1973]; circles — Tanasevitch et al. [2009]. Hatched area - Esyunin \& Efimik [1996]; Tanasevitch et al. [2009]. Abbreviations: AT — arctic tundra subzone; NHT — northern hypoarctic tundra subzone; SHT — southern hypoarctic tundra subzone, after Rebristaya [2013].

Карта. Точки и районы сборов на п-ове Ямал. Залитые области — сборы 2013-2014 гг.: WC — район на западном побережье; EC - район на восточном побережье. Крест - сборы А. Бабенко, 1994 г.; квадрат — Holm [1973]; круги — Tanasevitch et al. [2009]. Заштрихованная область - Esyunin \& Efimik [1996]; Tanasevitch et al. [2009]. Сокращения: АТ — подзона арктических тундр тундровой зоны; NHT - подзона северных гипоарктических тундр; SHT — подзона южный гипоарктических тундр по Ребристая [2013].

- sedge-grass-moss associations along a small rivers with Salix lanata \& S. phylicifolia stands, Rubus chamaemorus, Carex aquatilis, Arctagrostis latifolia, Sphagnum cuspidatum, Aulacomnium turgidum, etc.

4. Meadows:

- diverse herbs-moss communities on hills among swampy tundra with Festuca cryophila, Poa arctica, Dryas octopetala, Carex rariflora, Oxyria digyna, Polemonium boreale, Pleurozium schreberi, Dicranum majus, Hylocomium splendens, etc.;

- diverse herbs-moss meadows on high floodplains of rivers with Salix lanata, Festuca cryophila, Carex arctisibirica, Arctophila fulva, Antennaria arctica, Astragalus subpolaris, Polytrichum juniperinum, P. strictum, etc.;

- swampy Equisetum-sedge meadow communities on valley slopes with Equisetum arvense, Carex aquatilis, Calliergon sp., Polytrichum juniperum, P. strictum \& Racomitrium lanuginosum, etc.

Azonal communities - the plant communities that are not confined to a certain zone but distributed across many zones; in northern Yamal Peninsula, this type is represented by specific beach plant communities on seashore, marshlands and banks of $\mathrm{Ob}$ ' and SabettaYakha Rivers. The main part (80-90\%) of these communities are presented by bare sandy surface overgrown with grass and sedge vegetation Calamagrostis holmii, Carex rotundata, Deschampsia borealis, Poa alpigena, Alopecurus alpinum and sometimes with Eriophorum medium.

\section{Region and localities}

The present material was collected from 14 localities situated in the western coast of Yamal Peninsula (WC), and from 17 localities on its eastern coast (EC) in 2013-2014; in total, more than 60 sites were investigated. The majority of localities lied in the southern part of the arctic tundra subzone of tundra belt and only a few of them were in the hypoarctic tundra subzone; sensu Rebristaya [2013], see Map. A list of the locali- 
ties, their coordinates and abbreviations used in the following text are given below.

Western coast (WC 1-2) of northern Yamal, Cape

Kharasavey, 23.VIII.2014, leg. L.R.:

$\mathrm{WC} 1-71.295873^{\circ} \mathrm{N} 67.135860^{\circ} \mathrm{E}$

$\mathrm{WC} 2-71.268465^{\circ} \mathrm{N} 66.955659^{\circ} \mathrm{E}$

WC of northern Yamal, middle-flow of Sil-Yakha

River, 23.VIII.2014, leg. L.R.:

$\mathrm{WC} 3-71.163878^{\circ} \mathrm{N} 67.385128^{\circ} \mathrm{E}$

WC (4-6) of northern Yamal, mouth of Kharasavey

River, 23.VIII.2014, leg. L.R.:

$\mathrm{WC} 4-71.097452^{\circ} \mathrm{N} 66.870795^{\circ} \mathrm{E}$

$\mathrm{WC} 5-71.087943^{\circ} \mathrm{N} 66.810968^{\circ} \mathrm{E}$

$\mathrm{WC} 6-71.018381^{\circ} \mathrm{N} 67.036919^{\circ} \mathrm{E}$

WC (7-14) of northern Yamal, environs of Kruzenshtern Bay (= Guba Kruzenshterna) region, 22.VIII. 2014, leg. L.R.:

$\mathrm{WC7}-70.768722^{\circ} \mathrm{N} 67.471044^{\circ} \mathrm{E}$

$\mathrm{WC} 8-70.690405^{\circ} \mathrm{N} 67.371732^{\circ} \mathrm{E}$

WC9 $-70.648813^{\circ} \mathrm{N} 67.377769^{\circ} \mathrm{E}$

$\mathrm{WC} 10-70.630901^{\circ} \mathrm{N} 67.492567^{\circ} \mathrm{E}$

$\mathrm{WC} 11-70.603732^{\circ} \mathrm{N} 67.302487^{\circ} \mathrm{E}$

$\mathrm{WC} 12-70.561420^{\circ} \mathrm{N} 67.367152^{\circ} \mathrm{E}$

$\mathrm{WC} 13-70.503129^{\circ} \mathrm{N} 67.298986^{\circ} \mathrm{E}$

$\mathrm{WC} 14-70.473132^{\circ} \mathrm{N} 67.367152^{\circ} \mathrm{E}$

Eastern coast (EC 1-17) of northern Yamal, Ob'

River Bay (= Obskaya Guba), Tambey Bay region, $5.5-25 \mathrm{~km} \mathrm{~N}, \mathrm{~W}$ or S of Sabetta:

$\mathrm{EC} 1-71.495531^{\circ} \mathrm{N} 71.726795^{\circ} \mathrm{E}, 17 . \mathrm{VIII} .2014$, leg. V.O.

$\mathrm{EC} 2-71.435789^{\circ} \mathrm{N} 71.553767^{\circ} \mathrm{E}, 17-19 . \mathrm{VII}$

2013, leg. L.R. \& A.B.

$\mathrm{EC} 3-71.433194^{\circ} \mathrm{N} 71.564833^{\circ} \mathrm{E}, 19 . \mathrm{VIII} .2014$, leg. L.R.

$\mathrm{EC} 4-71.397929^{\circ} \mathrm{N} 71.840956^{\circ} \mathrm{E}, 19-21 . \mathrm{VII}$.

2013, leg. L.R. \& A.B.

EC5 - 71.280306 ${ }^{\circ} \mathrm{N} 71.542972^{\circ} \mathrm{E}, 19$. VIII.2014, leg. L.R.

EC6 - $71.272759^{\circ} \mathrm{N} 71.788439^{\circ} \mathrm{E}, 21-23 . \mathrm{VII}$.

2013, leg. L.R. \& A.B.; M.K.

$\mathrm{EC} 7-71.243583^{\circ} \mathrm{N} 71.696389^{\circ} \mathrm{E}, 15 . \mathrm{VIII} .2014$, leg. L.R.

$\mathrm{EC} 8-71.225917^{\circ} \mathrm{N} 71.512556^{\circ} \mathrm{E}, 19 . \mathrm{VIII} .2014$, leg. L.R.

EC9 - $71.224722^{\circ} \mathrm{N} 71.620278^{\circ} \mathrm{E}, 18$. VIII.2014, leg. L.R.

$\mathrm{EC} 10-71.192361^{\circ} \mathrm{N} 71.601167^{\circ} \mathrm{E}, 11 . \mathrm{VIII} .2014$,

leg. L.R. \& A.T.

EC1 $1-71.186278^{\circ} \mathrm{N} 71.588056^{\circ} \mathrm{E}, 11 . \mathrm{VIII} .2014$, leg. A.T.

$\mathrm{EC} 12-71.18308^{\circ} \mathrm{N} 71.69903^{\circ} \mathrm{E}, 11 . \mathrm{VIII} .2014$, leg. A.T.

$\mathrm{EC} 13-71.182778^{\circ} \mathrm{N} 71.852000^{\circ} \mathrm{E}, 15 . \mathrm{VIII} .2014$, leg. L.R.

EC14 - $71.228694^{\circ} \mathrm{N} 71.929417^{\circ} \mathrm{E}, 17 . \mathrm{VIII} .2014$, leg. L.R.

$\mathrm{EC} 15-71.232185^{\circ} \mathrm{N} 72.069636^{\circ} \mathrm{E}, 23-25 . \mathrm{VII}$.

2013, leg. L.R. \& A.B.

$\mathrm{EC} 16-71.112111^{\circ} \mathrm{N} 72.269189^{\circ} \mathrm{E}, 13 . \mathrm{VIII} .2014$, leg. L.R.
$\mathrm{EC} 17-71.090670^{\circ} \mathrm{N} 72.330783^{\circ} \mathrm{E}, 17-27 . \mathrm{VII}$. 2013, leg. L.R. \& A.B.

Northern coast (NC) of Yamal, delta Yakhadi-Yakha River, $72.8666^{\circ} \mathrm{N} 70.9333^{\circ} \mathrm{E}, 20-21 . V I I I .1994$, leg. An.B.

Each species account given below contains 'Remarks' in which a species distributional pattern is, as a rule, described in both regional (latitudinal) and zonal (meridional) terms. For example, a "Siberian-Nearctic arctic range" means that the species is distributed both in Siberia and in the Nearctic Region and occurs in the arctic zone.

The ecological characteristic, such as "eurytopic", "polytopic", "zonal", "intrazonal" and "azonal", are related to the territory of northern Yamal only; in other regions species ecotype characteristics may be different. The term "polyzonal" is used in its traditional sense, i.e., the species that inhabits all (or almost all) natural zones of the Palaearctic Region.

Abbreviations used in the text and table: Zonal, Intra, Azonal - zonal, intrazonal and azonal plant communities, respectively; A.B. - Alexander Bastrakov; A.T - Andrei Tanasevitch; An.B. - Anatoly Babenko; EC - the eastern coast of Yamal Peninsula; L.R. Leonid Rybalov; M.K. - Marina Khrisanova; NC the northern coast of Yamal Peninsula; V.O. — Vladimir Onufriev; WC — the western coast of Yamal Peninsula.

\section{A list of spiders of northern Yamal Penin-} sula

Families are listed in the alphabetical order; the number of species recorded is given in parentheses after the name.

\section{Fam. GNAPHOSIDAE (1)}

\section{Micaria constricta Emerton, 1894}

MATERIAL. Zonal. EC2: $3 \Im^{\top} \sigma^{\top}, 4$ oᄋ , lichen-moss-undershrub polygonal tundra on gentle slope of a brook valley with Cassiope tetragona, Equisetum arvense, Arctagrostis latifolia \& Polytrichum sp., sifting, by hands \& pitfall traps 17-19.VII.2013, leg. L.R. \& A.B.

Intra. EC2: $1 \sigma^{7}, 1$, swampy sedge-undershrub meadow on slope of valley with Equisetum arvense \& Carex aquatilis, sifting, 17.VII.2013, leg. L.R. \& A.B. EC4: $1 \sigma^{\top}$, lichen-moss-grass-undershrub tundra on river terrace with Cassiope tetragona, Equisetum arvense \& Arctagrostis latifolia, pitfall traps, 19-21.VII.2013, leg. L.R. \& A.B.

REMARKS. Holarctic arcto-boreal range. A polytopic species inhabiting both zonal and intrazonal plant communities.

Fam. LINYPHIIDAE (31)

Arcterigone pilifrons (L. Koch, 1879)

MATERIAL. Zonal. EC2: 1 q, lichen-moss-undershrub smallpolygonal tundra with Cassiope tetragona, Vaccinium vitis-idaea $\&$ Equisetum arvense, from tussocks of Arctagrostis latifolia, sifting, 18.VII.2013, leg. L.R. \& A.B. EC13: 1 \%, moss-sedge-grass 
polygonal tundra with Arctagrostis latifolia \& Carex arctisibirica, sifting, 15.VIII.2014, leg. L.R.

Intra. WC7: 1 , sedge-sphagnum swampy tundra near lake with Salix lanata \& Carex aquatilis, sifting, 22.VIII.2014, leg. L.R. WC9: 2 우, sedge-sphagnum swamp with Salix lanata, $S$. glauca, Carex arctisibirica \& C. aquatilis, sifting, 22.VIII.2014, leg. L.R. WC10: 1 , sedge-moss tundra on gentle slope to lake with Salix glauca stands, Arctagrostis latifolia \& Carex arctisibirica, sifting, 22.VIII.2014, leg. L.R. WC12: $2 \sigma^{7} \sigma^{7}$, sedge-sphagnum swampy tundra with Salix lanata, Polytrichum sp., \& Carex aquatilis, sifting, 22.VIII.2014, leg. L.R. EC2: 2 O $^{7} \sigma^{7}$, wet sedgemoss meadow on small stream terrace with Carex aquatilis \& $C$ arctisibirica, sifting \& pitfall traps, 17-19.VII.2013, leg. L.R. \& A.B. EC4: $1 \sigma^{7}$, moss-grass-sedge ribbon-shaped associations in depressions among polygonal tundra with Arctagrostis latifolia, Carex arctisibirica \& Pleurozium schreberi, sifting, 20.VII.2013, leg. L.R. \& A.B.; 1 ㅇ, lichen-moss-grass-undershrub tundra on river terrace with Cassiope tetragona, Equisetum arvense \& Arctagrostis latifolia, in grass tussocks, sifting, 20.VII.2013, leg. L.R. \& A.B EC5: 2 우, wet sedge-grass-moss-undershrub tundra along a small river with Salix lanata, S. phylicifolia, Cassiope tetragona, Carex aquatilis \& C. rariflora, sifting, 19. VIII.2014, leg. L.R. EC6: $1 \sigma^{7}$, diverse herbs-moss associations on hills among swampy tundra with Festuca cryophila, Poa arctica, Dryas octopetala, Carex rariflora, Oxyria digyna, Polemonium boreale, Dicranum majus \& Hylocomium splendens, sifting, 22.VII.2013, leg. L.R. \& A.B. EC7: $1 \sigma^{7}$, sphagnum-grass swamp near Sabetta-Yakha River bank with Carex aquatilis \& C. arctisibirica, sifting, 15.VIII.2014, leg. L.R. EC9: 1 + , sedge-grass-moss tundra with Salix glauca, $S$ phylicifolia, Eriophorum polystachion, E. media, Polygonum viviparum, Arctagrostis latifolia \& Carex arctisibirica, sifting, 18.VIII.2014, leg. L.R. EC10: 2 O $^{\top} \sigma^{7}, 5$ of, sedge-moss hummocky swamp with Carex aquatilis, $C$. rariflora, Comarum palustre, Eriophorum media, Aulacomnium turgidum, Calliergon richardsonii \& Mnium sp., sifting, 11.VIII.2014, leg. L.R. \& A.T. EC11: $2 \bigcirc^{7} \sigma^{2}, 13$ 우, diverse herbs-sedge-lichen-moss tundra near lake with Salix glauca stands, Carex aquatilis, Aulacomnium turgidum \& Calliergon richardsonii, sifting, 11.VIII.2014, leg. A.T. EC13: $1 \sigma^{7}, 1$ + , moss-sedge-undershrub tundra with Rubus chamaemorus, Carex arctisibirica \& Calamagrostis holmii, sifting, 15.VIII.2014, leg. L.R. EC15: $1 \bigcirc^{7}$, moss-grass-sedge ribbonshaped associations in depressions among polygonal tundra with Pleurozium schreberi, Arctagrostis latifolia, Carex arctisibirica \& Poa alpina, sifting, 26.VII.2013, leg. L.R. \& A.B. EC16: 1 ㅇ, Salix glauca \& S. reticulata stands on floodplain with Astragalus subpolaris, Carex arctisibirica, Festuca cryophila \& Peltigera sp., sifting, 13.VIII.2014, leg. L.R.; 2 우, Salix glauca stands on wet terrace with $S$. nummularia, Peltigera sp., Carex arctisibirica \& Alopecurus alpinus, sifting, 13.VIII.2014, leg. L.R. \& A.T.; 1 $\mathrm{O}^{7}$, Salix glauca stands on gentle slope of river terrace with Carex arctisibirica, Arctagrostis latifolia \& Eriophorum media, sifting, 13.VIII.2014, leg. A.T. EC17: 2 ㅇ, swampy diverse herbs-sedge regularly inundated meadow near lake with Arctagrostis latifolia, Carex aquatilis, C. arctisibirica, Eriophorum polystachion, E. medium, Comarum palustre \& Ranunculus pallasii, in hummocks of Aulacomnium palustre, A. turgidum \& Calliergon richardsonii, sifting, 24.VII.2013, leg. L.R. \& A.B.

REMARKS. Siberian-West Nearctic arctic range, west of Yamal Peninsula registered only from the Pechora Sea coast, Ugra Peninsula [Eskov, 1985]. An eurytopic species inhabiting almost all landscape elements and most of the plant communities. New record to Yamal Peninsula.

\section{Bathyphantes humilis (L. Koch, 1879)}

MATERIAL. Intra. EC2: $1 \sigma^{\text {tr }}$, wet sedge-moss meadow on small stream terrace with Carex aquatilis \& C. arctisibirica, sifting \& pitfall traps, 17-19.VII.2013, leg. L.R. \& A.B. EC6: 1 , diverse herbs-moss associations on hills among swampy tundra with Festuca cryophila, Poa arctica, Dryas octopetala, Carex rariflora, Oxyria digyna, Polemonium boreale, Dicranum majus \& Hylocomium splendens, sifting, 22.VII.2013, leg. L.R. \& A.B. EC8: 2 + , sedge-moss-undershrub tundra with Salix nummularia, Vaccinium uliginosum, V. vitis-idaea, Arctagrostis latifolia, Carex arctisibirica, C. aquatilis \& Polygonum bistorta, sifting, 19.VIII.2014, leg. L.R. EC11: 1 o, diverse herbs-sedge-lichenmoss tundra near lake with Salix glauca stands, Carex aquatilis, Aulacomnium turgidum \& Calliergon richardsonii, sifting,

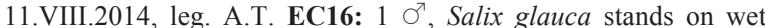
terrace with $S$. nummularia, Peltigera sp., Carex arctisibirica \& Alopecurus alpinus, sifting, 13.VIII.2014, leg. L.R. \& A.T. EC17: $2 \mathrm{O}^{2} \mathrm{O}^{7}, 1$, swampy diverse herbs-sedge regularly inundated meadow near lake with Arctagrostis latifolia, Carex aquatilis, C. arctisibirica, Eriophorum polystachion, E. medium, Comarum palustre \& Ranunculus pallasii, sifting \& pitfall traps, 23-25.VII.2013, leg. L.R. \& A.B.

Azonal. EC15: $1 \sigma^{7}$, grass meadow on sandy beach of $\mathrm{Ob}^{\text {' }}$ River Gulf with Deschampsia borealis, Poa alpigena, Alopecurus alpinus \& Eriophorum medium, pitfall traps, 17-25.VII.2013, leg. L.R. \& A.B.

REMARKS. Siberian range, west of Yamal Peninsula known up to base of Kanin Peninsula [Tanasevitch \& Koponen, 2007]. The species avoids zonal communities, a typical intrazonal species.

\section{Dactylopisthes video (Chamberlin et Ivie, 1947)}

MATERIAL. Azonal. EC15: 1 , grass meadow on sandy beach of Ob' River Gulf with Deschampsia borealis, Poa alpigena, Alopecurus alpinus \& Eriophorum medium, pitfall traps, 2325.VII.2013, leg. L.R. \& A.B.

REMARKS. East Palaearctic-West Nearctic range, west of Yamal Peninsula known up to the delta of Pechora River [Mazura, 2000]. Biotopic preferences are not clear.

\section{Diplocephalus barbiger (Roewer, 1955)}

MATERIAL. Zonal. EC15: 1 , moss-grass-sedge ribbonshaped associations in depressions among polygonal tundra with Pleurozium schreberi, Arctagrostis latifolia, Carex arctisibirica \& Poa alpina, sifting, 26.VII.2013, leg. L.R. \& A.B. EC15: 1 O" $^{\text {, }}$ sedge-moss-lichen tundra on gentle slopes of hills with Salix nummularia, Carex arctisibirica \& Rubus chamaemorus, sifting \& pitfall traps, 17-27.VII.2013, leg. L.R. \& A.B.

Intra. EC2: $1 \sigma^{7}, 1$, wet sedge-moss meadow on small stream terrace with Carex aquatilis \& C. arctisibirica, sifting \& pitfall traps, 17-19.VII.2013, leg. L.R. \& A.B. EC4: 1 \%, lichenmoss-grass-undershrub tundra on river terrace with Cassiope tetragona, Equisetum arvense \& Arctagrostis latifolia, in grass tussocks, sifting, 20.VII.2013, leg. L.R. \& A.B.

Azonal. EC6: 3 어, grass-sedge meadow on beach of SabettaYakha River with Calamagrostis holmii, Carex rotundata, sifting \& pitfall traps, 21-23.VII.2013, leg. L.R. \& A.B.

REMARKS. Siberian-Nearctic arctic range. West of Yamal Peninsula was registered in Vaygach Ins. [Holm, 1973; Eskov, 1985] and Novaya Zemlya [Dahl, 1928; Holm, 1973]. A polytopic species inhabiting most of landscape elements and plant communities.

\section{Erigone arctica palaearctica Braendegaard, 1934}

1973 Erigone arctica sibirica Kulczyński, 1908. - Holm: 82, misidentification.

MATERIAL. Zonal. EC2: $1 \sigma^{7}$, lichen-moss-undershrub polygonal tundra on gentle slope of a brook valley with Cassiope tetragona, Equisetum arvense, Arctagrostis latifolia \& Polytri- 
chum sp., sifting \& pitfall traps, 17-19.VII.2013, leg. L.R. \& A.B. EC15: $50 \bigcirc^{\top} \sigma^{7}, 30$ $+\circ$, sedge-moss-lichen tundra on gentle slopes of hills with Salix nummularia, Carex arctisibirica \& Rubus chamaemorus, sifting \& pitfall traps, 17-27.VII.2013, leg. L.R. \& A.B.

Intra. EC2: $9 \sigma^{\top} \sigma^{\top}, 3$ o , wet sedge-moss meadow on small stream terrace with Carex aquatilis \& C. arctisibirica, sifting \& pitfall traps, 17-19.VII.2013, leg. L.R. \& A.B. EC3: $1 \sigma^{7}, 1$, wet sedge-grass-moss tundra among stream with Deschampsia borealis \& Luzula confusa, sifting, 19.VIII.2014, leg. L.R. EC4: $4 \sigma^{7} \sigma^{7}$ 1 o, lichen-moss-grass-undershrub tundra on river terrace with Cassiope tetragona, Equisetum arvense \& Arctagrostis latifolia pitfall traps, 19-21.VII.2013, leg. L.R. \& A.B.; $1 \sigma^{\top}, 1$ \%, lichenmoss-grass-undershrub tundra on river terrace with Cassiope tetragona, Equisetum arvense \& Arctagrostis latifolia, in grass tussocks, sifting, 20.VII.2013, leg. L.R. \& A.B.; $12 \sigma^{7} \sigma^{7}, 6$ of , mossgrass-sedge ribbon-shaped associations in depressions among polygonal tundra with Arctagrostis latifolia, Carex arctisibirica \& Pleurozium schreberi, in dry grass tussocks, sifting, 19-21.VII.2013, leg. L.R. \& A.B. EC5: $1 \sigma^{7}$, wet sedge-grass-moss-undershrub tundra along a small river with Salix lanata, S. phylicifolia, Cassiope tetragona, Carex aquatilis \& C. rariflora, sifting, 19. VIII.2014, leg. L.R. EC6: $6 \sigma^{7} \sigma^{7}, 4$ 0 , swampy grass meadows in former riverbed depressions in floodplain of Sabetta-Yakha River with Eriophorum media, Calamagrostis holmii \& Carex aquatilis, sifting \& pitfall traps, 21-23.VII.2013, leg. L.R. \& A.B.

Azonal. EC4: $60 \sigma^{\top} \sigma^{\top} \&$ \& , sedge-grass sandy beach associations along a small river with Eriophorum media, Calamagrostis holmii, Carex rotundata \& Polytrichum sp., sifting \& pitfall traps, 19-21.VII.2013, leg. L.R. \& A.B.; $4 \sigma^{\top} \sigma^{\top}, 2$, 2 , sandy beach along a small river with single grass tussocks, Eriophorum media, $\mathrm{Ca}$ lamagrostis holmii \& Carex rotundata, sifting \& pitfall traps, 1921.VII.2013, leg. L.R. \& A.B. EC15: $2 \sigma^{7} \sigma^{7}, 2$, 2 , grass meadow on sandy beach of Ob' River Gulf with Deschampsia borealis, Poa alpigena, Alopecurus alpinus \& Eriophorum medium, pitfall traps, 17-25.VII.2013, leg. L.R. \& A.B. EC17: $3 \sigma^{\top} \sigma^{\top}, 3$ 우, swampy moss associations in ravine with Eriophorum polystachion, E. medium, Carex aquatilis, Calliergon richardsonii, Aulacomnium palustre \& Sphagnum squarrosum, sifting \& pitfall traps, 23 25.VII.2013, leg. L.R. \& A.B.

REMARKS. With a high probability, this species was misidentified on the basis of a female as Erigone arctica sibirica from the western coast of northern Yamal $\left(72^{\circ} 18^{\prime} \mathrm{N} 68^{\circ} 42^{\prime} \mathrm{E}\right.$, see Map) [Holm, 1973]. The presence of both sexes of Erigone a. palaearctica recorded from neighbouring territories is evidence that the record should be referred to the latter species.

East European arctic range. An eurytopic species inhabiting all landscape elements and plant communities and attaining a high abundance.

\section{Erigone psychrophila Thorell, 1872}

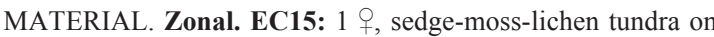
gentle slopes of hills with Salix nummularia, Carex arctisibirica \& Rubus chamaemorus, sifting \& pitfall traps, 17-27.VII.2013, leg. L.R. \& A.B.

Intra. EC2: 2 ㅇ, wet sedge-moss meadow on small stream terrace with Carex aquatilis \& C. arctisibirica, sifting \& pitfall traps, 17-19.VII.2013, leg. L.R. \& A.B. EC6: 1 ' $\sigma^{7}$, swampy grass meadows in former riverbed depressions in floodplain of SabettaYakha River with Eriophorum media, Calamagrostis holmii \& Carex aquatilis, sifting \& pitfall traps, 21-23.VII.2013, leg. L.R. \& A.B. EC17: $1 \sigma^{7}, 1$, swampy diverse herbs-sedge regularly inundated meadow near lake with Arctagrostis latifolia, Carex aquatilis, C. arctisibirica, Eriophorum polystachion, E. medium, Comarum palustre \& Ranunculus pallasii, sifting \& pitfall traps, 23-25.VII.2013, leg. L.R. \& A.B.

Azonal. EC6: $60 \sigma^{\top} \sigma^{\top} \&$ \& $9+$, grass-sedge meadow on beach of Sabetta-Yakha River with Calamagrostis holmii, Carex rotundata, pitfall traps, 21-23.VII.2013, leg. L.R. \& A.B. EC17: 1 \%, swampy moss associations in ravine with Eriophorum polystachion, E. medium, Carex aquatilis, Calliergon richardsonii, Aulacomnium palustre \& Sphagnum squarrosum, sifting \& pitfall traps, 2325.VII.2013, leg. L.R. \& A.B. NC: $1 \sigma^{7}, 3$ क+ , northern coast of Yamal Peninsula, delta Yakhadi-Yakha River, marshland with Puccinella sp., driftwood, leg. An.B.

REMARKS. Holarctic arctic range. A polytopic species inhabiting most of landscape elements and plant communities.

\section{Erigone remota L. Koch, 1869}

MATERIAL. Intra. EC6: 1 , swampy grass meadows in former riverbed depressions in floodplain of Sabetta-Yakha River with Eriophorum media, Calamagrostis holmii \& Carex aquatilis, sifting \& pitfall traps, 21-23.VII.2013, leg. L.R. \& A.B.

REMARKS. Palaearctic arcto-alpine range. Biotopic preferences are not clear; probably an intrazonal species.

\section{Erigone tirolensis L. Koch, 1872}

MATERIAL. Intra. EC12: $1 \sigma^{\top}, 2$, $\odot$, gentle slopes in depressions among diverse herbs-moss-lichen-undershrub tundra with Cassiope tetragona, Equisetum arvense, Carex arctisibirica, Dryas octopetala, Festuca cryophila, Hedysarum arcticum \& Arctagrostis latifolia, sifting, 11.VIII.2014, leg. A.T.; 2 0 , grass-moss meadow in valley with Polytrichum juniperinum, P. strictum, Pleurozium schreberi, Dryas octopetala, Equisetum arvense \& Festuca cryophila, sifting, 11.VIII.2014, leg. L.R.

REMARKS. Holarctic arcto-alpine range. Biotopic preferences are not clear; probably an intrazonal species.

\section{Gibothorax tchernovi Eskov, 1989}

MATERIAL. Zonal. EC2: lichen-moss-undershrub small-polygonal tundra with Cassiope tetragona, Vaccinium vitis-idaea \& Equisetum arvense, from tussocks of Arctagrostis latifolia, sifting, 18.VII.2013, leg. L.R. \& A.B.

Intra. EC2: $1 \sigma^{7}$, wet sedge-moss meadow on small stream terrace with Carex aquatilis \& C. arctisibirica, sifting \& pitfall traps, 17-19.VII.2013, leg. L.R. \& A.B. EC4: 15 ○ $\sigma^{\top}$, lichenmoss-grass-undershrub tundra on river terrace with Cassiope tetragona, Equisetum arvense \& Arctagrostis latifolia, in grass tussocks, sifting, 20.VII.2013, leg. L.R. \& A.B. EC6: 1 ○', diverse herbs-moss associations on hills among swampy tundra with Festuca cryophila, Poa arctica, Dryas octopetala, Carex rariflora, Oxyria digyna, Polemonium boreale, Dicranum majus \& Hylocomium splendens, sifting, 22.VII.2013, leg. L.R. \& A.B.; $1 \sigma^{7}$, diverse herbs-moss associations on hills among swampy tundra with Festuca cryophila, Poa arctica, Dryas octopetala, Carex rariflora, Oxyria digyna \& Polemonium boreale, sifting, 21-23.VII.2013, leg. L.R. \& A.B.; 1 ऽ', swampy grass meadows in former riverbed depressions in floodplain of Sabetta-Yakha River with Eriophorum media, Calamagrostis holmii \& Carex aquatilis, sifting \& pitfall traps, 21-23.VII.2013, leg. L.R. \& A.B. EC17: $5 \sigma^{7} \sigma^{7}, 1$, swampy diverse herbs-sedge regularly inundated meadow near lake with Arctagrostis latifolia, Carex aquatilis, C. arctisibirica, Eriophorum polystachion, E. medium, Comarum palustre \& Ranunculus pallasii, sifting \& pitfall traps, 23-25.VII.2013, leg. L.R. \& A.B.

REMARKS. Siberian arctic range. West of Yamal Peninsula known from Dolgy Ins., Pechora Sea only [Marusik, Eskov, 2009]. A polytopic species inhabiting most of landscape elements but preferring intrazonal plant communities. 


\section{Halorates holmgreni (Thorell, 1871)}

1973 Collinsia holmgreni (Thorell, 1871). - Holm: 81.

MATERIAL. Zonal. EC3: $1 \sigma^{7}, 1$, moss-lichen-undershrub polygonal tundra on gentle slope to small river with Cassiope tetragona \& Carex arctisibirica, sifting, 19.VIII.2014, leg. L.R. EC13: 2 + ${ }^{\circ}$, lichen-moss-undershrub tundra with Cassiope tetragona, Eriophorum russeolum, Calamagrostis holmii, Poa arctica \& Carex arctisibirica, sifting, 15.VIII.2014, leg. L.R. EC6: $2 \sigma^{7} \sigma^{7}$ dry lichen tundra, pitfall traps, 12-15.IX.2013, leg. M.K.

Intra. EC2: 9 우, wet sedge-moss meadow on small stream terrace with Carex aquatilis \& C. arctisibirica, sifting \& pitfall traps, 17-19.VII.2013, leg. L.R. \& A.B.; 4 우, swampy sedgeundershrub meadow on slope of valley with Equisetum arvense, Carex aquatilis, Calliergon richardsonii \& Aulacomnium turgidum, sifting, 18.VII.2013, leg. L.R. \& A.B. EC3: $5 \sigma^{7} \sigma^{7}, 6$ 우, wet sedgegrass-moss tundra among stream with Deschampsia borealis \& Luzula confusa, sifting, 19.VIII.2014, leg. L.R. EC4: $8 \sigma^{7} \sigma^{7}, 10$ 우, lichen-moss-grass-undershrub tundra on river terrace with Cassiope tetragona, Equisetum arvense \& Arctagrostis latifolia, pitfall traps, 19-21.VII.2013, leg. L.R. \& A.B.; 3 90 , moss-grass-sedge ribbonshaped associations in depressions among polygonal tundra with Arctagrostis latifolia, Carex arctisibirica \& Pleurozium schreberi, sifting, 19-21.VII.2013, leg. L.R. \& A.B.; 3 o+ , sedge-moss-undershrub tundra with Equisetum arvense, Polytrichum juniperinum, Alopecurus alpinus, Carex arctisibirica \& Polygonum viviparum, sifting, 17.VIII.2014, leg. L.R. EC11: $2 \sigma^{\top} \sigma^{7}, 6$ O+ , diverse herbssedge-lichen-moss tundra near lake with Salix glauca stands, Carex aquatilis, Aulacomnium turgidum \& Calliergon richardsonii, sifting, 11.VIII.2014, leg. A.T. EC12: $2 \sigma^{\top} \sigma^{\top}, 6$ 우, gentle slopes in depressions among diverse herbs-moss-lichen-undershrub tundra with Cassiope tetragona, Equisetum arvense, Carex arctisibirica, Dryas octopetala, Festuca cryophila, Hedysarum arcticum \& Arctagrostis latifolia, sifting, 11.VIII.2014, leg. A.T. EC16: $18 \Im^{\top} \sigma^{\top}, 12$ 우, Salix glauca stands on gentle slope of river terrace with Carex arctisibirica, Arctagrostis latifolia \& Eriophorum media, sifting, 13.VIII.2014, leg. A.T. EC17: 1 q, swampy moss associations in ravine with Eriophorum polystachion, E. medium, Carex aquatilis, Calliergon richardsonii, Aulacomnium palustre \& Sphagnum squarrosum, sifting \& pitfall traps, 23-25.VII.2013, leg. L.R. \& A.B.; 2 $\sigma^{7} \sigma^{\top}, 1$, , diverse herbs-moss tundra on slope with Calamagrostis holmii, Artemisia borealis, Arctagrostis latifolia, Festuca cryophila, Polemonium borealis, Tanacetum bipinnatum \& Polytrichum sp., sifting \& pitfall traps, 23-25.VII.2013, leg. L.R. \& A.B.; 2 qO, diverse herbs-moss tundra on slope with Calamagrostis holmii, Artemisia borealis, Arctagrostis latifolia, Festuca cryophila, Polemonium borealis, Tanacetum bipinnatum \& Polytrichum sp., sifting, 24.VII.2013, leg. L.R. \& A.B.

Azonal. EC4: $50 \sigma^{\top} \sigma^{\top} \&$ $\circ \circ$, sedge-grass sandy beach associations along a small river with Eriophorum media, Calamagrostis holmii, Carex rotundata \& Polytrichum sp., sifting \& pitfall traps, 19-21.VII.2013, leg. L.R. \& A.B.; $1 \sigma^{7}, 1$ \%, sandy beach along a small river with single grass tussocks, Eriophorum media, Calamagrostis holmii \& Carex rotundata, sifting, 20.VII.2013, leg. L.R. \& A.B. EC17: $1 \sigma^{7}, 13$ $+\circ$, swampy moss associations in ravine with Eriophorum polystachion, E. medium, Carex aquatilis, Calliergon richardsonii, Aulacomnium palustre \& Sphagnum squarrosum, sifting \& pitfall traps, 23-25.VII.2013, leg. L.R. \& A.B.

REMARKS. Holarctic arcto-alpine range. An eurytopic species inhabiting all landscape elements and plant communities and attaining a high abundance there. This species was hitherto recorded from the western coast of northern Yamal $\left(72^{\circ} 18^{\prime} \mathrm{N} 68^{\circ} 42^{\prime} \mathrm{E}\right)$ [Holm, 1973], see Map.

\section{Halorates spetsbergensis (Thorell, 1872)}

MATERIAL. Zonal. EC15: $10 \bigcirc^{7} \sigma^{7}$, sedge-moss-lichen tundra on gentle slopes of hills with Salix nummularia, Carex arctisibirica \& Rubus chamaemorus, sifting \& pitfall traps, 1727.VII.2013, leg. L.R. \& A.B.
Intra. EC16: 1 을 Salix glauca \& S. reticulata stands on floodplain with Astragalus subpolaris, Carex arctisibirica, Festuca cryophila \& Peltigera sp., sifting, 13.VIII.2014, leg. L.R.

REMARKS. Holarctic arctic range. A typical zonal species.

\section{Hilaira glacialis (Thorell, 1871)}

MATERIAL. Zonal. WC1: $1 \sigma^{\text {r }}$, lichen-moss-undershrub polygonal tundra on slope to Kara Sea with Salix nummularia, $S$. polaris, Pleurozium schreberi, Dicranum sp., and with spots of bare ground, covered partly with crustose lichens, sifting, 23.VIII.2014, leg. L.R. EC2: 1 क, lichen-moss-undershrub smallpolygonal tundra with Cassiope tetragona, Vaccinium vitis-idaea \& Equisetum arvense, from tussocks of Arctagrostis latifolia, sifting, 18.VII.2013, leg. L.R. \& A.B. EC6: $4 \bigcirc^{7} \bigcirc^{7}, 2$ 9 , dry lichen tundra, pitfall traps, 12-15.IX.2013, leg. M.K. EC7: 1 \%, Ledumsedge-undershrub tundra near Sabetta-Yakha River bank with Ledum decumbens, Cassiope tetragona, Carex arctisibirica, Vaccinium vitis-idaea \& Arctagrostis latifolia, sifting, 15.VIII.2014, leg. L.R. $1 \sigma^{\top}, 1$, sedge-grass-undershrub tundra near Sabetta-Yakha River bank with Cassiope tetragona, Calamagrostis holmii, Carex arctisibirica \& Deschampsia borealis, sifting, 15.VIII.2014, leg. L.R. EC13: $1 \sigma^{\top}, 1$, lichen-moss-undershrub tundra with Cassiope tetragona, Eriophorum russeolum, Calamagrostis holmii, Poa arctica \& Carex arctisibirica, sifting, 15.VIII.2014, leg. L.R.; 3 $\sigma^{\top} \sigma^{7}, 3$ 우, moss-sedge-grass polygonal tundra with Arctagrostis latifolia \& Carex arctisibirica, sifting, 15.VIII.2014, leg. L.R. EC15: $1 \sigma^{7}, 1$, sedge-moss-lichen tundra on gentle slopes of hills with Salix nummularia, Carex arctisibirica \& Rubus chamaemorus, sifting \& pitfall traps, 17-27.VII.2013, leg. L.R. \& A.B.

Intra. WC5: 2 $\sigma^{7}$, sedge-grass-moss tundra near Kara seashore, with Polytrichum sp., Pleurozium schreberi, Arctagrostis latifolia \& Deschampsia borealis, sifting, 23.VIII.2014, leg. L.R. EC4: $1 \sigma^{7}, 2$ 우, moss-grass-sedge ribbon-shaped associations in depressions among polygonal tundra with Arctagrostis latifolia, Carex arctisibirica \& Pleurozium schreberi, sifting, 19-

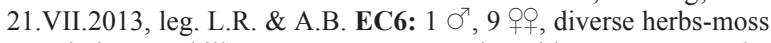
associations on hills among swampy tundra with Festuca cryophila, Poa arctica, Dryas octopetala, Carex rariflora, Oxyria digyna, Polemonium boreale, Dicranum majus \& Hylocomium splendens, sifting, 22.VII.2013, leg. L.R. \& A.B.; 3 O$^{\top} \sigma^{\top}, 11$ of, diverse herbs-moss associations on hills among swampy tundra with Festuca cryophila, Poa arctica, Dryas octopetala, Carex rariflora, Oxyria digyna \& Polemonium boreale, sifting, 21-23.VII.2013, leg. L.R. \& A.B. EC8: $1 \sigma^{7}, 1$, sedge-moss-undershrub tundra with Salix nummularia, Vaccinium uliginosum, V. vitis-idaea, Arctagrostis latifolia, Carex arctisibirica, Carex aquatilis \& Polygonum bistorta, sifting, 19.VIII.2014, leg. L.R. EC13: $3 \sigma^{\top} \sigma^{\top}, 1$, moss-sedge-undershrub tundra with Rubus chamaemorus, Carex arctisibirica \& Calamagrostis holmii, sifting, 15.VIII.2014, leg. L.R. EC15: $5 \Im^{7} \Im^{7}, 11$ +Q, moss-grass-sedge ribbon-shaped associations in depressions among polygonal tundra with Pleurozium schreberi, Arctagrostis latifolia, Carex arctisibirica \& Poa alpi-

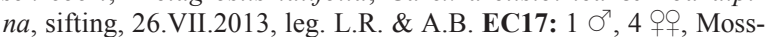
grass-sedge ribbon-shaped associations in depressions among polygonal tundra with Pleurozium schreberi, Arctagrostis latifolia, Carex arctisibirica \& Poa alpina, sifting, 23-25.VII.2013, leg. L.R. \& A.B.

REMARKS. Siberian arcto-boreo-montane range. A polytopic species inhabiting most of landscape elements and plant communities and attaining a high abundance.

\section{Hilaira incondita (L. Koch, 1879)}

MATERIAL. Intra. EC2: $17 \sigma^{\top} \sigma^{\top}, 4$ oo, wet sedge-moss meadow on small stream terrace with Carex aquatilis \& C. arctisibirica, sifting \& pitfall traps, 17-19.VII.2013, leg. L.R. \& A.B. EC4: 12 +, , lichen-moss-grass-undershrub tundra on river terrace 
with Cassiope tetragona, Equisetum arvense \& Arctagrostis latifolia, in grass tussocks, sifting, 19-21.VII.2013, leg. L.R. \& A.B. EC6: $3 \bigcirc^{\top} \sigma^{7}, 12$ 우, swampy grass meadows in former riverbed depressions in floodplain of Sabetta-Yakha River with Eriophorum media, Calamagrostis holmii \& Carex aquatilis, sifting \& pitfall traps, 21-23.VII.2013, leg. L.R. \& A.B. EC6: $1 \sigma^{7}, 1$ \%, wet mosssedge tundra, pitfall traps, 12-15.IX.2013, leg. M.K. EC14: 1 , wet sedge-moss-undershrub tundra with Equisetum arvense, Polytrichum juniperinum, Alopecurus alpinus, Carex arctisibirica, Polygonum viviparum, Calliergon richardsonii \& Aulacomnium turgidum, sifting, 17.VIII.2014, leg. L.R.; $2 \bigcirc^{\top} \sigma^{\top}, 2$ 우, Salix glauca stands on gentle slope of river terrace with Carex arctisibirica, Arctagrostis latifolia \& Eriophorum media, sifting, 13.VIII.2014, leg. A.T.

Azonal. EC4: $1 \checkmark^{\top}$, sedge-grass sandy beach associations along a small river with Eriophorum media, Calamagrostis holmii, Carex rotundata \& Polytrichum sp., sifting \& pitfall traps, 1921.VII.2013, leg. L.R. \& A.B. EC6: $1 \sigma^{7}, 4$ 우, grass-sedge meadow on beach of Sabetta-Yakha River with Calamagrostis holmii, Carex rotundata, sifting \& pitfall traps, 21-23.VII.2013, leg. L.R. \& A.B. EC15: $1 \sigma^{\top}, 2$, 2 , grass meadow on sandy beach of $\mathrm{Ob}$ River Gulf with Deschampsia borealis, Poa alpigena, Alopecurus alpinus \& Eriophorum medium, pitfall traps, 17-25.VII.2013, leg. L.R. \& A.B.

REMARKS. Siberian-Nearctic arcto-boreal range. A typical intrazonal species avoiding zonal communities.

\section{Hilaira nivalis Holm, 1937}

MATERIAL. Zonal. WC14: $1 \sigma^{\gamma}, 3$ $+{ }_{+}$, moss-sedge tundra with Salix glauca \& Carex arctisibirica, sifting, 22.VIII.2014, leg. L.R. EC5: $2 \sigma^{\top} \sigma^{\top}, 6$ 우, moss-lichen-undershrub polygonal tundra with Arctagrostis latifolia, Carex arctisibirica, sifting, 26.VII.2013.

Intra. WC10: 1 , sedge-moss tundra on gentle slope to lake with Salix glauca stands, Arctagrostis latifolia \& Carex arctisibiri$c a$, sifting, 22.VIII.2014, leg. L.R. EC4: $2 \sigma^{\top} \sigma^{7}$, lichen-moss-grassundershrub tundra on river terrace with Cassiope tetragona, Equisetum arvense \& Arctagrostis latifolia, in grass tussocks, sifting, 1921.VII.2013, leg. L.R. \& A.B. EC5: 1 丁 , 4 우, wet sedge-grassmoss-undershrub tundra along a small river with Salix lanata, $S$ phylicifolia, Cassiope tetragona, Carex aquatilis \& C. rariflora, sifting, 19. VIII.2014, leg. L.R. EC6: $1 \sigma^{\top}, 1$, diverse herbs-moss associations on hills among swampy tundra with Festuca cryophila, Poa arctica, Dryas octopetala, Carex rariflora, Oxyria digyna \& Polemonium boreale, sifting, 21-23.VII.2013, leg. L.R. \& A.B.; 2 $\sigma^{\top} \sigma^{\top}, 2$ 우, wet moss-sedge tundra, pitfall traps, 12-15.IX.2013, leg. M.K. EC7: 1 , sphagnum-grass swamp near Sabetta-Yakha River bank with Carex aquatilis \& C. arctisibirica, sifting, 15.VIII.2014, leg. L.R. EC9: $2 \sigma^{7} \sigma^{7}, 4$ $9+$, sedge-grass-moss tundra with Salix glauca, S. phylicifolia, Eriophorum polystachion, E. media, Polygonum viviparum, Arctagrostis latifolia \& Carex arctisibirica, sifting, 18.VIII.2014, leg. L.R. EC10: 5 o, sedge-moss hummocky swamp with Carex aquatilis, C. rariflora, Comarum palustre, Eriophorum media, Aulacomnium turgidum, Calliergon richardsonii \& Mnium sp., sifting, 11.VIII.2014, leg. L.R. \& A.T. EC11: $3 \sigma^{\top} \sigma^{\top}, 3$ 우, diverse herbs-sedge-lichen-moss tundra near lake with Salix glauca stands, Carex aquatilis, Aulacomnium turgidum \& Calliergon richardsonii, sifting, 11.VIII.2014, leg. A.T. EC13: 1 \% , 3 우, mosssedge-undershrub tundra with Rubus chamaemorus, Carex arctisibirica \& Calamagrostis holmii, sifting, 15.VIII.2014, leg. L.R. EC16: $3 \sigma^{\top} \sigma^{\top}, 7$, 90 , Salix glauca stands on wet terrace with $S$. nummularia, Peltigera sp., Carex arctisibirica \& Alopecurus alpinus, sifting, 13.VIII.2014, leg. L.R. \& A.T.; 3 O $^{7} \sigma^{7}, 10$ 우, Salix glauca stands on gentle slope of river terrace with Carex arctisibirica, Arctagrostis latifolia \& Eriophorum media, sifting, 13.VIII.2014, leg. A.T.; $1 \sigma^{\top}$ 2 +o, Salix glauca \& S. reticulata stands on floodplain with Astragalus subpolaris, Carex arctisibirica, Festuca cryophila \& Peltigera sp., sifting, 13.VIII.2014, leg. L.R.

Azonal. NC: $1 \sigma^{7}, 3$ 90 , northern coast of Yamal Peninsula, delta of Yakhadi-Yakha River, marshland with Puccinella sp., driftwood, 20-21.VIII.1994, leg. An.B.
REMARKS. West Siberian arctic range. An eurytopic species inhabiting all landscape elements and plant communities and attaining a high abundance. New record to Yamal Peninsula.

\section{Hilaira proletaria (L. Koch, 1879)}

MATERIAL. Intra. EC6: $2 \sigma^{7} \sigma^{7}, 6$ 우, wet moss-sedge tundra, pitfall traps, 12-15.IX.2013, leg. M.K. EC10: $2 \sigma^{\top} \sigma^{\top}, 5$, 5 , sedge-moss hummocky swamp with Carex aquatilis, C. rariflora, Comarum palustre, Eriophorum media, Aulacomnium turgidum, Calliergon richardsonii \& Mnium sp., sifting, 11.VIII.2014, leg. L.R. \& A.T. EC16: $1 \sigma^{r}, 1$, Salix glauca \& S. reticulata stands on floodplain with Astragalus subpolaris, Carex arctisibirica, Festuca cryophila \& Peltigera sp., sifting, 13.VIII.2014, leg. L.R. EC17: 1 , swampy diverse herbs-sedge regularly inundated meadow near lake with Arctagrostis latifolia, Carex aquatilis, C. arctisibirica, Eriophorum polystachion, E. medium, Comarum palustre \& Ranunculus pallasii, sifting \& pitfall traps, 23-25.VII.2013, leg. L.R. \& A.B.

REMARKS. Siberian-West Nearctic arcto-boreal range. West of Yamal Peninsula recorded from the Pechora Sea coast, Ugra Peninsula only [Eskov, 1985]. A typical intrazonal species avoiding zonal communities.

\section{Hybauchenidium aquilonare (L. Koch, 1879)}

MATERIAL. Zonal. WC14: 1 , moss-sedge tundra with Salix glauca \& Carex arctisibirica, sifting, 22.VIII.2014, leg. L.R.

Intra. WC10: $1+$, sedge-moss tundra on gentle slope to lake with Salix glauca stands, Arctagrostis latifolia \& Carex arctisibirica, sifting, 22.VIII.2014, leg. L.R. WC11: 1 \%, sedge-moss tundra with Salix glauca stands \& Carex arctisibirica, sifting, 22.VIII.2014, leg. L.R.

REMARKS. Siberian-West Nearctic arcto-boreal range. West of Yamal Peninsula known up to the delta of Pechora River [Mazura, 2000]. Biotopic preferences are not clear.

\section{Masikia indistincta (Kulczyński, 1908)}

MATERIAL. Zonal. EC2: $1 \sigma^{\text {, }}$, lichen-moss-undershrub polygonal tundra on gentle slope of a brook valley with Cassiope tetragona, Equisetum arvense, Arctgrostis latifolia \& Polytrichum sp., sifting \& pitfall traps, 17-19.VII.2013, leg. L.R. \& A.B.; 3 우, lichen-moss-undershrub small-polygonal tundra with Cassiope tetragona, Vaccinium vitis-idaea \& Equisetum arvense, from tussocks of Arctagrostis latifolia, sifting \& pitfall traps, 18.VII.2013, leg. L.R. \& A.B. EC3: 1 \%, moss-lichen-undershrub polygonal tundra on gentle slope to small river with Cassiope tetragona \& Carex arctisibirica, sifting, 19.VIII.2014, leg. L.R.

Intra. WC2: 2 क , sedge-sphagnum-undershrub tundra with Salix glauca and spots of Pleurozium schreberi, Aulacomnium sp. on regularly inundated marchland, sifting, 23.VIII.2014, leg. L.R. WC3: $2 \Im^{7}, 2$ 우, sedge-moss tundra near small river with Polytrichum sp., Sphagnum sp. \& Pleurozium schreberi, sifting, 23.VIII.2014, leg. L.R. WC6: 3 우, sedge-sphagnum-undershrub swampy tundra on bank of Kharasavey River with Salix lanata \& Carex aquatilis, sifting, 23.VIII.2014, leg. L.R. WC12: 1 , sedgesphagnum swampy tundra with Salix lanata, Polytrichum sp., \& Carex aquatilis, sifting, 22.VIII.2014, leg. L.R. EC2: $5 \sigma^{\top} \sigma^{7}, 27$ + , wet sedge-moss meadow on small stream terrace with Carex aquatilis \& C. arctisibirica, sifting \& pitfall traps, 17-19.VII.2013, leg. L.R. \& A.B. EC3: 2 +⿱ stream with Deschampsia borealis \& Luzula confusa, sifting, 
19.VIII.2014, leg. L.R. EC4: 5 O $^{\top} \sigma^{\top}, 7$ 우, moss-grass-sedge ribbon-shaped associations in depressions among polygonal tundra with Arctagrostis latifolia, Carex arctisibirica \& Pleurozium schreberi, sifting, 19-21.VII.2013, leg. L.R. \& A.B. $1 \bigcirc^{7}$, lichen-moss-grassundershrub tundra on river terrace with Cassiope tetragona, Equisetum arvense \& Arctagrostis latifolia, in grass tussocks, sifting, 1921.VII.2013, leg. L.R. \& A.B. EC5: 1 t, wet sedge-grass-mossundershrub tundra along a small river with Salix lanata, S. phylicifolia, Cassiope tetragona, Carex aquatilis \& C. rariflora, sifting, 19. VIII.2014, leg. L.R. EC6: $1 \sigma^{7}$, swampy grass meadows in former riverbed depressions in floodplain of Sabetta-Yakha River with Eriophorum media, Calamagrostis holmii \& Carex aquatilis, sifting \& pitfall traps, 21-23.VII.2013, leg. L.R. \& A.B.; 7 우, diverse herbsmoss associations on hills among swampy tundra with Festuca cryophila, Poa arctica, Dryas octopetala, Carex rariflora, Oxyria digyna, Polemonium boreale, Dicranum majus \& Hylocomium splendens, sifting, , 21-23.VII.2013, leg. L.R. \& A.B.; 3 우어, diverse herbs-moss associations on hills among swampy tundra with Festuca cryophila, Poa arctica, Dryas octopetala, Carex rariflora, Oxyria digyna \& Polemonium boreale, sifting, 21-23.VII.2013, leg. L.R. \& A.B. EC7: 4 우, sphagnum-grass swamp near Sabetta-Yakha River bank with Carex aquatilis \& C. arctisibirica, sifting, 15.VIII.2014, leg. L.R. EC9: $3 \sigma^{7} \sigma^{7}, 1$, sedge-grass-moss tundra with Salix glauca, S. phylicifolia, Eriophorum polystachion, E. media, Polygonum viviparum, Arctagrostis latifolia \& Carex arctisibirica, sifting, 18.VIII.2014, leg. L.R. EC10: $4 \bigcirc^{7} \sigma^{7}, 24$ PO, sedgemoss hummocky swamp with Carex aquatilis, C. rariflora, Comarum palustre, Eriophorum media, Aulacomnium turgidum, Calliergon richardsonii \& Mnium sp., sifting, 11.VIII.2014, leg. L.R. \& A.T. EC11: $3 \sigma^{7} \sigma^{7}, 5$ 우, diverse herbs-sedge-lichen-moss tundra near lake with Salix glauca stands, Carex aquatilis, Aulacomnium turgidum \& Calliergon richardsonii, sifting, 11.VIII.2014, leg. A.T. EC13: $2 O^{7} \sigma^{7}, 5$ OO , moss-sedge-undershrub tundra with Rubus chamaemorus, Carex arctisibirica \& Calamagrostis holmii, sifting, 15.VIII.2014, leg. L.R. EC15: $8 \sigma^{7} \sigma^{7}, 9$, 9 , moss-grass-sedge ribbon-shaped associations in depressions among polygonal tundra with Pleurozium schreberi, Arctagrostis latifolia, Carex arctisibirica \& Poa alpina, sifting, 26.VII.2013, leg. L.R. \& A.B. EC16: $3 \bigcirc^{7} \sigma^{7}, 3$ OO, Salix glauca \& S. reticulata stands on floodplain with Astragalus subpolaris, Carex arctisibirica, Festuca cryophila \& Peltigera sp., sifting, 13.VIII.2014, leg. L.R. EC17: 5 o $^{\prime \prime} \sigma^{7}, 24$ 우, swampy diverse herbs-sedge regularly inundated meadow near lake with Arctagrostis latifolia, Carex aquatilis, C. arctisibirica, Eriophorum polystachion, E. medium, Comarum palustre \& Ranunculus pallasii, sifting \& pitfall traps, 23-25.VII.2013, leg. L.R. \& A.B.

Azonal. EC4: $1 O^{7}, 1$, sedge-grass sandy beach associations along a small river with Eriophorum media, Calamagrostis holmii, Carex rotundata \& Polytrichum sp., sifting \& pitfall traps, 1921.VII.2013, leg. L.R. \& A.B.; 1 O", sandy beach along a small river with single grass tussocks, Eriophorum media, Calamagrostis holmii \& Carex rotundata, sifting \& pitfall traps, 19-21.VII.2013, leg. L.R. \& A.B.

REMARKS. Siberian-Nearctic arcto-boreal range. An eurytopic species inhabiting all landscape elements and plant communities and attaining a high abundance.

\section{Mecynargus tundricola Eskov, 1988}

MATERIAL. Zonal. WC14: $1 \sigma^{7}, 3$ ㅇ, moss-sedge tundra with Salix glauca \& Carex arctisibirica, sifting, 22.VIII.2014, leg. L.R. EC1: $1 \sigma^{7}$, grass-moss \& moss-lichen tundra, 17.VIII.2014, leg. V.O. EC2: 1 \%, lichen-moss-undershrub polygonal tundra on gentle slope of a brook valley with Cassiope tetragona, Equisetum arvense, Arctagrostis latifolia \& Polytrichum sp., sifting \& pitfall traps, 17-19.VII.2013, leg. L.R. \& A.B.; 2 우, lichen-moss-undershrub small-polygonal tundra with Cassiope tetragona, Vaccinium vitis-idaea \& Equisetum arvense, sifting, 18.VII.2013, leg. L.R. \& A.B. EC7: 2 +P, sedge-grass-undershrub tundra near Sabetta-Yakha River bank with Cassiope tetragona, Calamagrostis holmii, Carex arctisibirica \& Deschampsia borealis, sifting, 15.VIII.2014, leg. L.R. EC13: 2 우, lichen-moss-undershrub tundra with Cassiope tetragona, Eriophorum russeolum, Calamagrostis holmii, Poa arctica \& Carex arctisibirica, sifting, 15.VIII.2014, leg. L.R.; 1 $\mathrm{O}^{\top}$, sedge-moss-lichen tundra on gentle slopes of hills with Salix nummularia, Carex arctisibirica \& Rubus chamaemorus, sifting \& pitfall traps, 17-27.VII.2013, leg. L.R. \& A.B.

Intra. WC10: 3 우, sedge-moss tundra on gentle slope to lake with Salix glauca stands, Arctagrostis latifolia \& Carex arctisibiri$c a$, sifting, 22.VIII.2014, leg. L.R. WC11: 1 , sedge-moss tundra with Salix glauca stands \& Carex arctisibirica, sifting, 22.VIII.2014, leg. L.R. WC12: $30^{7} \sigma^{7}$, sedge-sphagnum swampy tundra with Salix lanata, Polytrichum sp., \& Carex aquatilis, sifting, 22.VIII.2014, leg. L.R. EC2: $2 \sigma^{7} \sigma^{7}, 1$, wet sedge-moss meadow on small stream terrace with Carex aquatilis \& C. arctisibirica, sifting \& pitfall traps, 17-19.VII.2013, leg. L.R. \& A.B. EC4: $20^{7} \sigma^{7}, 2$ 우, mossgrass-sedge ribbon-shaped associations in depressions among polygonal tundra with Arctagrostis latifolia, Carex arctisibirica \& Pleurozium schreberi, sifting, 19-21.VII.2013, leg. L.R. \& A.B.; 1 , lichen-moss-grass-undershrub tundra on river terrace with Cassiope tetragona, Equisetum arvense \& Arctagrostis latifolia, pitfall traps, 19-21.VII.2013, leg. L.R. \& A.B. EC6: 5 ऽ $^{\top} \sigma^{7}, 5$ 우, diverse herbsmoss associations on hills among swampy tundra with Festuca cryophila, Poa arctica, Dryas octopetala, Carex rariflora, Oxyria digyna \& Polemonium boreale, sifting, 21-23.VII.2013, leg. L.R. \& A.B.; 1 , swampy grass meadows in former riverbed depressions in floodplain of Sabetta-Yakha River with Eriophorum media, $\mathrm{Ca}$ lamagrostis holmii \& Carex aquatilis, sifting \& pitfall traps, 2123.VII.2013, leg. L.R. \& A.B. EC8: 1 ơ $^{2}$, sedge-moss-undershrub tundra with Salix nummularia, Vaccinium uliginosum, V. vitis-idaea, Arctagrostis latifolia, Carex arctisibirica, Carex aquatilis \& Polygonum bistorta, sifting, 19.VIII.2014, leg. L.R. EC13: 3 ○ $^{7} \sigma^{7}, 3$ 우, moss-sedge-undershrub tundra with Rubus chamaemorus, Carex arctisibirica \& Calamagrostis holmii, sifting, 15.VIII.2014, leg. L.R. EC15: $11 \sigma^{7} \sigma^{7}, 22$ 우, moss-grass-sedge ribbon-shaped associations in depressions among polygonal tundra with Pleurozium schreberi, Arctagrostis latifolia, Carex arctisibirica \& Poa alpina, sifting, 26.VII.2013, leg. L.R. \& A.B. EC17: 1 , swampy diverse herbssedge regularly inundated meadow near lake with Arctagrostis latifolia, Carex aquatilis, C. arctisibirica, Eriophorum polystachion, E. medium, Comarum palustre \& Ranunculus pallasii, in hummocks of Dicranum flexicaule \& Aulacomnium palustre, sifting, 24.VII.2013, leg. L.R. \& A.B

REMARKS. Siberian arcto-boreal range. West of Yamal Peninsula known from Dolgy Ins., Pechora Sea only [Marusik, Eskov, 2009]. An eurytopic species inhabiting almost all landscape elements and plant communities and attaining a high abundance. New record to Yamal Peninsula.

\section{Mughiphantes sobrius (Thorell, 1872)}

MATERIAL. Zonal. EC7: $1 \sigma^{7}$, Ledum-sedge-undershrub tundra near Sabetta-Yakha River bank with Ledum decumbens, Cassiope tetragona, Carex arctisibirica, Vaccinium vitis-idaea \& Arctagrostis latifolia, sifting, 15.VIII.2014, leg. L.R.

Intra. EC12: 2 ㅇ, gentle slopes in depressions among diverse herbs-moss-lichen-undershrub tundra with Cassiope tetragona, Equisetum arvense, Carex arctisibirica, Dryas octopetala, Festuca cryophila, Hedysarum arcticum \& Arctagrostis latifolia, sifting, 11.VIII.2014, leg. A.T.

REMARKS. Palaearctic arctic range. Biotopic preferences are not clear. New record to Yamal Peninsula.

\section{Oreoneta leviceps (L. Koch, 1879)}

MATERIAL. Intra. WC12: $1 \sigma^{7}, 2$ ㅇ, sedge-sphagnum swampy tundra with Salix lanata, Polytrichum sp., \& Carex aquatilis, sifting, 22.VIII.2014, leg. L.R.

REMARKS. Siberian-Nearctic arcto-boreal range. Biotopic preferences are not clear. Probably intrazonal species. 


\section{Paraglyphesis polaris Eskov, 1991}

MATERIAL. Intra. WC6: $1 \bigcirc^{7}, 1$, sedge-sphagnum-undershrub swampy tundra on bank of Kharasavey River with Salix lanata \& Carex aquatilis, sifting, 23.VIII.2014, leg. L.R. EC10: 1 +, sedge-moss hummocky swamp with Carex aquatilis, C. rariflora, Comarum palustre, Eriophorum media, Aulacomnium turgidum, Calliergon richardsonii \& Mnium sp., sifting, 11.VIII.2014, leg. L.R. \& A.T.

REMARKS. Siberian arcto-boreal range. Biotopic preferences are not clear; probably, an intrazonal species. Yamal Peninsula is the westernmost limit of its known distribution. New record to Yamal Peninsula.

\section{Pelecopsis parallela (Wider, 1834)}

MATERIAL. Intra. WC6: 1 , sedge-sphagnum-undershrub swampy tundra on bank of Kharasavey River with Salix lanata \& Carex aquatilis, sifting, 23.VIII.2014, leg. L.R. EC2: 2 우, wet sedge-moss meadow on small stream terrace with Carex aquatilis \& C. arctisibirica, sifting \& pitfall traps, 17-19.VII.2013, leg. L.R. \& A.B. EC9: $1 \mathrm{O}^{7}, 1$, , sedge-grass-moss tundra with Salix glauca, S. phylicifolia, Eriophorum polystachion, E. media, Polygonum viviparum, Arctagrostis latifolia \& Carex arctisibirica, sifting, 18.VIII.2014, leg. L.R. EC15: 1 + , moss-grass-sedge ribbon-shaped associations in depressions among polygonal tundra with Pleurozium schreberi, Arctagrostis latifolia, Carex arctisibirica \& Poa alpina, sifting, 26.VII.2013, leg. L.R. \& A.B. EC16: $3 \sigma^{7} \sigma^{2}, 1$, Salix glauca stands on gentle slope of river terrace with Carex arctisibirica, Arctagrostis latifolia \& Eriophorum media, sifting, 13.VIII.2014, leg. A.T

REMARKS. Palaearctic polyzonal range. Inhabiting most intrazonal plant communities.

Perro polaris (Eskov, 1986)

MATERIAL. Zonal. EC15: 5 +q, sedge-moss-lichen tundra on gentle slopes of hills with Salix nummularia, Carex arctisibirica \& Rubus chamaemorus, sifting \& pitfall traps, 23-25.VII.2013, leg. L.R. \& A.B.

Intra. EC4: 3 qo, lichen-moss-grass-undershrub tundra on river terrace with Cassiope tetragona, Equisetum arvense \& Arctagrostis latifolia, sifting \& pitfall traps, 19-21.VII.2013, leg. L.R. \& A.B.

REMARKS. Siberian-West Nearctic arcto-boreal range. Biotopic preferences are not clear. New record to Yamal Peninsula.

\section{Semljicola alticola $($ Holm, 1950)}

MATERIAL. Zonal. EC2: $1 \sigma^{7}, 1$, lichen-moss-undershrub small-polygonal tundra with Cassiope tetragona, Vaccinium vitisidaea \& Equisetum arvense, from tussocks of Arctagrostis latifolia, sifting, 18.VII.2013, leg. L.R. \& A.B. EC7: $1 \sigma^{7}$, sedge-grassundershrub tundra near Sabetta-Yakha River bank with Cassiope tetragona, Calamagrostis holmii, Carex arctisibirica \& Deschampsia borealis, sifting, 15.VIII.2014, leg. L.R.

Intra. WC2: 1 , sedge-sphagnum-undershrub tundra with Salix glauca and spots of Pleurozium schreberi, Aulacomnium sp. on regularly inundated marchland, sifting, 23.VIII.2014, leg. L.R. WC6: $1 \sigma^{\text {T }, ~} 1$, , sedge-sphagnum-undershrub swampy tundra on bank of Kharasavey River with Salix lanata \& Carex aquatilis, sifting, 23.VIII.2014, leg. L.R. EC3: 1 \%', 1 , wet sedge-grass-moss tundra among stream with Deschampsia borealis \& Luzula confusa, sifting, 19.VIII.2014, leg. L.R. EC11: 2 $\sigma^{\top} \sigma^{\top}, 5$ 90 , diverse herbs-sedge-lichen-moss tundra near lake with Salix glauca stands, Carex aquatilis, Aulacomnium turgidum \& Calliergon richardsonii, sifting, 11.VIII.2014, leg. A.T.
EC16: 1 ๑ 26 오, Salix glauca stands on wet terrace with $S$. nummularia, Peltigera sp., Carex arctisibirica \& Alopecurus alpinus, sifting, 13.VIII.2014, leg. L.R. \& A.T.; 1 ऽ , 22 우, Salix glauca stands on gentle slope of river terrace with Carex arctisibirica, Arctagrostis latifolia \& Eriophorum media, sifting, 13.VIII.2014, leg. A.T.

Azonal. EC15: 2 웅 grass meadow on sandy beach of $\mathrm{Ob}^{\prime}$ River Gulf with Deschampsia borealis, Poa alpigena, Alopecurus alpinus \& Eriophorum medium, pitfall traps, 17-25.VII.2013, leg. L.R. \& A.B.

REMARKS. Fennoscandian-Siberian arcto-boreal range. An eurytopic species inhabiting almost all landscape elements and plant communities and attaining a high abundance.

\section{Semljicola arcticus (Eskov, 1989)}

MATERIAL. Zonal. WC1: $1 \sigma^{7}$, lichen-moss-undershrub polygonal tundra on slope to Kara Sea with Salix nummularia $S$. polaris, Pleurozium schreberi, Dicranum sp., and with spots of bare ground, covered partly with crustose lichens, sifting, 23.VIII.2014, leg. L.R. EC2: 1 o, lichen-moss-undershrub polygonal tundra on gentle slope of a brook valley with Cassiope tetragona, Equisetum arvense, Arctagrostis latifolia \& Polytrichum sp., sifting \& pitfall traps, 17-19.VII.2013, leg. L.R. \& A.B.; 1 \%', lichen-moss-undershrub small-polygonal tundra with Cassiope tetragona, Vaccinium vitis-idaea \& Equisetum arvense, sifting, 18.VII.2013, leg. L.R. \& A.B. EC7: $1 \sigma^{7}$, sedge-grass-undershrub tundra near Sabetta-Yakha River bank with Cassiope tetragona, Calamagrostis holmii, Carex arctisibirica \& Deschampsia borealis, sifting, 15.VIII.2014, leg. L.R.; 1 +, Ledum-sedge-undershrub tundra near Sabetta-Yakha River bank with Ledum decumbens, Cassiope tetragona, Carex arctisibirica, Vaccinium vitis-idaea \& Arctagrostis latifolia, sifting, 15.VIII.2014, leg. L.R. EC13: $1 \sigma^{\top}, 1$, moss-sedge-grass polygonal tundra with Arctagrostis latifolia \& Carex arctisibirica, sifting, 15.VIII.2014, leg. L.R. EC17: 1 q, lichen-moss-undershrub polygonal tundra with Cassiope tetragona, Vaccinium vitis-idaea \& Carex arctisibirica, sifting, 23-25.VII.2013, leg. L.R. \& A.B.

Intra. EC4: $1 \sigma^{7}$, moss-grass-sedge ribbon-shaped associations in depressions among polygonal tundra with Arctagrostis latifolia, Carex arctisibirica \& Pleurozium schreberi, sifting, 1921.VII.2013, leg. L.R. \& A.B. EC5: $1 \sigma^{7}$, wet sedge-grass-mossundershrub tundra along a small river with Salix lanata, S. phylicifolia, Cassiope tetragona, Carex aquatilis \& C. rariflora, sifting, 19.VIII.2014, leg. L.R. EC6: $1 \sigma^{r}, 2$ 우, diverse herbs-moss associations on hills among swampy tundra with Festuca cryophila, Poa arctica, Dryas octopetala, Carex rariflora, Oxyria digyna, Polemonium boreale, Dicranum majus \& Hylocomium splendens, sifting, 21-23.VII.2013, leg. L.R. \& A.B. EC8: 1 ○', sedge-mossundershrub tundra with Salix nummularia, Vaccinium uliginosum, V. vitis-idaea, Arctagrostis latifolia, Carex arctisibirica, C. aquatilis \& Polygonum bistorta, sifting, 19.VIII.2014, leg. L.R. EC10: $3 \sigma^{\top} \sigma^{7}, 2$ 우, sedge-moss hummocky swamp with Carex aquatilis, C. rariflora, Comarum palustre, Eriophorum media, Aulacomnium turgidum, Calliergon richardsonii \& Mnium sp., sifting, 11.VIII.2014, leg. L.R. \& A.T. EC12: 2 of, gentle slopes in depressions among diverse herbs-moss-lichen-undershrub tundra with Cassiope tetragona, Equisetum arvense, Carex arctisibirica, Dryas octopetala, Festuca cryophila, Hedysarum arcticum \& Arctagrostis latifolia, sifting, 11.VIII.2014, leg. A.T. EC15: $7 \sigma^{7} \sigma^{7}, 5$ 0 , moss-grass-sedge ribbon-shaped associations in depressions among polygonal tundra with Pleurozium schreberi, Arctagrostis latifolia, Carex arctisibirica \& Poa alpina, sifting, 26.VII.2013, leg. L.R. \& A.B. EC16: $4 \sigma^{\top} \sigma^{7}$, Salix glauca stands on gentle slope of river terrace with Carex arctisibirica, Arctagrostis latifolia \& Eriophorum media, sifting, 13.VIII.2014, leg. A.T.

REMARKS. Siberian arctic range. West of Yamal Peninsula known from the Pechora Sea coast, Ugra Peninsula and from the northern coast of Vaygach Ins. [Eskov, 1985]. An eurytopic species inhabiting almost all landscape elements and plant communities. 


\section{Semljicola barbiger (L. Koch, 1879)}

MATERIAL. Zonal. WC14: 3 † , moss-sedge tundra with Salix glauca \& Carex arctisibirica, sifting, 22.VIII.2014, leg. L.R.

Intra. WC2: 1 , sedge-sphagnum-undershrub tundra with Salix glauca and spots of Pleurozium schreberi, Aulacomnium sp. on regularly inundated marchland, sifting, 23.VIII.2014, leg. L.R. WC11: 1 , sedge-moss tundra with Salix glauca stands \& Carex arctisibirica, sifting, 22.VIII.2014, leg. L.R. WC12: 1 \%, sedgesphagnum swampy tundra with Salix lanata, Polytrichum sp., \& Carex aquatilis, sifting, 22.VIII.2014, leg. L.R. EC2: 15 of, we sedge-moss meadow on small stream terrace with Carex aquatilis \& C. arctisibirica, sifting \& pitfall traps, 17-19.VII.2013, leg. L.R. \& A.B. EC5: 1 ㅇ, wet sedge-grass-moss-undershrub tundra along a small river with Salix lanata, S. phylicifolia, Cassiope tetragona, Carex aquatilis \& C. rariflora, sifting, 19. VIII.2014, leg. L.R EC6: 1 ऊ, wet moss-sedge tundra, pitfall traps, 12-15.IX.2013, leg. M.K. EC9: $1 \sigma^{7}, 5$ $\circ{ }_{+}$, sedge-grass-moss tundra with Salix glauca, S. phylicifolia, Eriophorum polystachion, E. media, Polygonum viviparum, Arctagrostis latifolia \& Carex arctisibiri$c a$, sifting, 18.VIII.2014, leg. L.R. EC10: $1 \sigma^{7}, 3$ of, sedge-moss hummocky swamp with Carex aquatilis, C. rariflora, Comarum palustre, Eriophorum media, Aulacomnium turgidum, Calliergon richardsonii \& Mnium sp., sifting, 11.VIII.2014, leg. L.R. \& A.T. EC16: $6 \sigma^{\top} \sigma^{\top}, 13$ 우, Salix glauca stands on wet terrace with $S$. nummularia, Peltigera sp., Carex arctisibirica \& Alopecurus alpinus, sifting, 13.VIII.2014, leg. L.R. \& A.T. EC17: $1 \sigma^{7}, 1$, mossgrass-sedge ribbon-shaped associations in depressions among polygonal tundra with Pleurozium schreberi, Arctagrostis latifolia Carex arctisibirica \& Poa alpina, sifting, 23-25.VII.2013, leg. L.R. \& A.B.

REMARKS. Siberian-Nearctic arcto-boreo-montane range. West of Yamal Peninsula known up to the Pechora River reaches [Tanasevitch, Koponen, 2007]. A polytopic species inhabiting almost all landscape elements and plant communities.

\section{Semljicola simplex (Kulczyński, 1908)}

MATERIAL. Zonal. EC15: 1 ア , 25 우, sedge-moss-lichen tundra on gentle slopes of hills with Salix nummularia, Carex arctisibirica \& Rubus chamaemorus, sifting \& pitfall traps, 1727.VII.2013, leg. L.R. \& A.B.

Intra. WC10: 1 , , sedge-moss tundra on gentle slope to lake with Salix glauca stands, Arctagrostis latifolia \& Carex arctisibirica, sifting, 22.VIII.2014, leg. L.R. WC11: $1 \sigma^{7}, 2$ + 0 , sedge-moss tundra with Salix glauca stands \& Carex arctisibirica, sifting, 22.VIII.2014, leg. L.R. EC2: 1 q, wet sedge-moss meadow on small stream terrace with Carex aquatilis \& C. arctisibirica, sifting \& pitfall traps, 17-19.VII.2013, leg. L.R. \& A.B.; 2 +中, swampy sedge-undershrub meadow on slope of valley with Equisetum arvense, Carex aquatilis, Calliergon richardsonii \& Aulacomnium turgidum, sifting, 18.VII.2013, leg. L.R. \& A.B. EC4: 1 ○', 2 우, moss-grass-sedge ribbon-shaped associations in depressions among polygonal tundra with Arctagrostis latifolia, Carex arctisibirica \& Pleurozium schreberi, sifting, 19-21.VII.2013, leg. L.R. \& A.B.; 7 $\bigcirc \mathrm{Q}$, lichen-moss-grass-undershrub tundra on river terrace with Cassiope tetragona, Equisetum arvense \& Arctagrostis latifolia, in grass tussocks, sifting, 19-21.VII.2013, leg. L.R. \& A.B. EC5: 1 , wet sedge-grass-moss-undershrub tundra along a small river with Salix lanata, S. phylicifolia, Cassiope tetragona, Carex aquatilis \& C. rariflora, sifting, 19. VIII.2014, leg. L.R. EC6: 4 우, diverse herbs-moss associations on hills among swampy tundra with Festuca cryophila, Poa arctica, Dryas octopetala, Carex rariflora, Oxyria digyna, Polemonium boreale, Dicranum majus \& Hylocomium splendens, sifting, 21-23.VII.2013, leg. L.R. \& A.B. EC10: 1 \%, sedge-moss hummocky swamp with Carex aquatilis, C. rariflora, Comarum palustre, Eriophorum media, Aulacomnium turgidum, Calliergon richardsonii \& Mnium sp., sifting, 11.VIII.2014, leg. L.R. \& A.T. EC12: 1 , gentle slopes in depressions among diverse herbs-moss-lichen-undershrub tundra with Cassiope tet- ragona, Equisetum arvense, Carex arctisibirica, Dryas octopetala, Festuca cryophila, Hedysarum arcticum \& Arctagrostis latifolia, sifting, 11.VIII.2014, leg. A.T. EC17: 2 \%, swampy diverse herbs-sedge regularly inundated meadow near lake with Arctagrostis latifolia, Carex aquatilis, C. arctisibirica, Eriophorum polystachion, E. medium, Comarum palustre \& Ranunculus pallasii, in hummocks of Aulacomnium palustre, Aulacomnium turgidum \& Calliergon richardsonii, sifting, 24.VII.2013, leg. L.R. \& A.B.

REMARKS. Siberian arcto-alpine range. An eurytopic species inhabiting almost all landscape elements and most of plant communities. Yamal Peninsula is the westernmost limit of its known distribution. New record to Yamal Peninsula.

\section{Silometopoides pampia (Chamberlin, 1948)}

MATERIAL. Intra. WC6: 1 , sedge-sphagnum-undershrub swampy tundra on bank of Kharasavey River with Salix lanata \& Carex aquatilis, sifting, 23.VIII.2014, leg. L.R. EC7: 1 , sphagnum-grass swamp near Sabetta-Yakha River bank with Carex aquatilis \& C. arctisibirica, sifting, 15.VIII.2014, leg. L.R. EC9: $1{ }^{7}$, 3 우, sedge-grass-moss tundra with Salix glauca, S. phylicifolia, Eriophorum polystachion, E. media, Polygonum viviparum, Arctagrostis latifolia \& Carex arctisibirica, sifting, 18.VIII.2014, leg. L.R. EC10: $1 \sigma^{\top}, 1$ \%, sedge-moss hummocky swamp with Carex aquatilis, C. rariflora, Comarum palustre, Eriophorum media, Aulacomnium turgidum, Calliergon richardsonii \& Mnium sp., sifting, 11.VIII.2014, leg. L.R. \& A.T. EC11: 1 ㅇ, diverse herbssedge-lichen-moss tundra near lake with Salix glauca stands, Carex aquatilis, Aulacomnium turgidum \& Calliergon richardsonii, sifting, 11.VIII.2014, leg. A.T. EC16: 1 + , Salix glauca stands on gentle slope of river terrace with Carex arctisibirica, Arctagrostis latifolia \& Eriophorum media, sifting, 13.VIII.2014, leg. A.T.; 1 + Salix glauca \& S. reticulata stands on floodplain with Astragalus subpolaris, Carex arctisibirica, Festuca cryophila \& Peltigera sp., sifting, 13.VIII.2014, leg. L.R. EC17: $2 \sigma^{7} \sigma^{7}, 4$ of, swampy diverse herbs-sedge regularly inundated meadow near lake with Arctagrostis latifolia, Carex aquatilis, C. arctisibirica, Eriophorum polystachion, E. medium, Comarum palustre \& Ranunculus pallasii, sifting \& pitfall traps, 23-25.VII.2013, leg. L.R. \& A.B.

TYPE MATERIAL EXAMINED. Silometopoides sphagnicola: All types from Putorana Plateau are examined; $1 \sigma^{7}$, RUSSIA, Yamal-Nenets Autonomous Region, Shchyuchya River, mouth of Tanlova-Yakha River, 13.VII.1979, leg. T. Andreeva; 1 ; , Taimyr Autonomous Region, middle flow of Pyasina River, Tareya, polygonal tundra, 5.VIII.1972, leg. Yu. Chernov; $5 \sigma^{7} \sigma^{7}, 19$ 우, Evenk Autonomous Region, Taimura River (left tributary of Nizhnyaya Tunguska River), mouth of Chambe River, boggy Larix taiga, 1823.VIII.1983, leg. K. Eskov; 2 우, Buryat Autonomous Republic, upper Vitim River, $40 \mathrm{~km}$ upstream of Zaza River mouth, boggy depression with Betula middendorfi in Larix taiga, 20.VIII.1983, leg. V. Zherikhin; $1 \sigma^{\top}$, Yakutia (Sakha Republic), Aldan River, Khandyga, 1.VII.1984, leg. A. Ryvkin; 3 of, Kempendyai River (right tributary of Vilyui River), $40 \mathrm{~km}$ upstream of Kempendyai Village, Sphagnum-Aulacomium bog, 7.VIII.1988, leg. K. Eskov; 2 $\sigma^{\top} \sigma^{\top}, 1$, Chita Area, Kyra District, Sokhonda Nature Reserve, Khentei Mts., Ingoda River, $1350 \mathrm{~m}$ a.s.1., dwarfish Alnus fruti-cosa thickets with green mosses, 8-9.VI.1991, leg. D. Logunov; $1 \sigma^{7}$, Amur Area, environs of Zeya Town, Sphagnum bog, 1-1l.IX.1979,

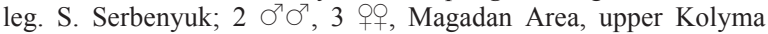
River, Sibit-Tyellakh, taiga belt of Bolshoi Annachag Mt. Range, small Carex swamp in boggy Larix taiga, 3.IX.1984, leg. K. Eskov \& Yu. Marusik. All from ZMMU.

COMPARATIVE MATERIAL EXAMINED. Silometopoides pampia: $1 \sigma^{\top}$, CANADA, Northwest Territories, Ellesmere Island, Alexandra Fjord, 15-26. VII. 1991, leg. T. Rossolimo; 7 q0 (as Minyriolus p.), RUSSIA, Chukotka Autonomous Region, upper reaches of Bolshaya Osinovaya River, Chosenia forest, litter, 13.VII.1989, leg. Yu. Marusik; $18 \sigma^{\top} \sigma^{\top}, 39$ 우, environs of Lavrentiya $\left(65.583491^{\circ} \mathrm{N} 171.010092^{\circ} \mathrm{W}\right)$, VII.2013, leg. A. Tanasevitch, O. Makarova \& A. Babenko; $1 \sigma^{\top}$, environs of Lavrentiya, Utaveem 
River, IX.1991, leg. N. Vekhov;1 9 , env. of Anadyr, 26.VI.1988; leg. Yu. Marusik; $3 \sigma^{\top} \sigma^{\top}, 2$ River (source of Amguema River), 66 $55^{\circ}$ N, $178^{\circ} 30^{\prime}$ E, Salix and Alnus fruticosa bushes on sandy beech, 12.VIII.1988, leg. Yu. Marusik; $2 \sigma^{\top} \sigma^{7}$ (as Minyriolus p.), northwestern coast of Kresta Gulf, floodland, Salix bushes with mosses, 28.VII.1988, leg. Yu. Marusik; 1 ๑, 6 우 (as Minyriolus p.), Kresta Gulf, 4.VII.1988, leg. Yu. Marusik; $1 \Im^{7}$ (as Minyriolus p.), Kresta Gulf, env. of Konergino, 3-5.VII.1988, leg. Yu. Marusik; $1 \sigma^{7}, 6$ of, env. of Konergino, tundra on southern slopes, mosses, 4.VII.1988, leg. Yu. Marusik; $1 \sigma^{7}$ (as Minyriolus p.), NW coast of Kresta Gulf, floodplain, Salix stands, mosses, 28.VII.1988, leg. Yu. Marusik; 1 q (as Minyriolus p.), Amguema River near Amguema Village $\left(67^{\circ} 03^{\prime} \mathrm{N}\right.$ 178 56'W), Sphagnum-Carex bog, 22.VIII.1988, leg. Yu. Marusik; $1 \sigma^{7}$ (as Minyriolus p.), Chaun Bay, delta of Chaun River, summer, 1986, leg. A. Ryabukhin; $1 \sigma^{\top}, 8$ 우 (as Minyriolus p.), mouth of Chaun River, 10-20.VII.1982, leg. I. Obutenkov; 3 o $\sigma^{7}, 2$ 우 (as Minyriolus p.), $64 \mathrm{~km} \mathrm{~N}$ of Egvekinot, Salix \& Betula stands with dry mosses, 18.VII.1988, leg. Yu. Marusik; 4 우 (as Minyriolus p.), $10 \mathrm{~km}$ of Egvekinot, swamp, among spring, mosses, 19.VII.1988, leg. Yu. Marusik; 7 우, Wrangel Island, middle Neizvestnaya River, dwarfish Salix with mosses and Carex, 1-23.VIII.1983, leg. O. Khruleva; $1 \sigma^{7}, 1$, same locality, river terrace, dwarfish Salix \& Dryas with mosses 27.VI.1983, leg. O. Khruleva; $2 \sigma^{7} \sigma^{7}, 1$, upper reaches of Gusinaya River, 1984, leg. O. Khruleva. All from ZMMU.

Silometopoides sphagnicola Eskov et Marusik, 1992: 1 7", Chukotka Autonomous Region, Chegitun River, 18-30.VIII.1992, leg. M. Berezin (new locality); 1 $\sigma^{\top}, 1$, Evenk Autonomous Region, Taimura River (left tributary of Nizhnyaya Tunguska River), mouth of Chambe River, boggy Larix taiga, 18-23.VIII.1983, leg. K. Eskov; $1 \sigma^{7}$ (as S. pampia, misidentification, reexamined by A. Tanasevitch, III.2015), Amurskaya Area, Norskiy Nature Reserve, Burunda River near Ozyornyi Rill mouth, 27.IX.2004, leg. A. Ryvkin; 1 , Norskiy Nature Reserve, Nora River basin, 2 $\mathrm{km}$ up-stream of Mt. Gryashchinskaya, mosses and plant debris on small open swamp on high flood-plain, 22.VIII.2004, leg. A. Ryvkin; $1+$ (as S . pampia, misidentification, reexamined by A. Tanasevitch, III.2015), Selemdzha River basin, floodplain of Aldikon River near Norsk, 5.IX.2004, leg. A. Ryvkin; $2 \sigma^{\top} \sigma^{7}$, Irkutsk Area, Zhigalogo District, pipeline Kovykta - Zhigalovo, floodplain meadow, VIII. 2006, leg. L. Rybalov (new locality). All from ZMMU.

REMARKS. The finding of Silometopoides pampia in northern Yamal was completely unexpected. As was earlier argued, the distribution of this species in the Palaearctic Region should be restricted to Chukotka, whereas entire Siberia (including southern Yamal) is inhabited by S. sphagnicola, a very closely related species [Eskov \& Marusik, 1992; Tanasevitch, 2006]. We have revised all the available type, comparative and newly collected materials of both species. All samples labelled as S. sphagnicola were proved to be correctly identified. Only a few individuals from Amur Area [Tanasevitch, 2006] and Chukotka, Chegitun River (unpublished) determined earlier as $S$. pampia proved to be misidentified and should actually be referred to $S$. sphagnicola (see above). The only female paratype of $S$. sphagnicola collected from the delta of Yana River (Kazachie) was not available for checking out. Thus, it appears that the population of $S$. pampia in northern Yamal is a small enclave of the species of which the main part of its distribution area lies in Arctic North America and Chukotka. S. sphagnicola has been recorded from eastern Chukotka for the first time, and therefore both S. sphagnicola and S. pampia are sympatric there.

DISTRIBUTION: Northern Yamal, Chukotka, Arctic North America. A typical intrazonal species avoiding zonal communities. New record to Yamal Peninsula.

\section{Tarsiphantes latithorax Strand, 1905}

MATERIAL. Zonal. EC2: $1 \sigma^{\gamma}, 3$ ㅇ, lichen-moss-undershrub polygonal tundra on gentle slope of a brook valley with Cassiope tetragona, Equisetum arvense, Arctagrostis latifolia \& Polytrichum sp., in dry grass tussocks, sifting, 18.VII.2013, leg. L.R. \& A.B.

Intra. EC12: $1 \sigma^{r}, 6$, 6 , gentle slopes in depressions among diverse herbs-moss-lichen-undershrub tundra with Cassiope tetragona, Equisetum arvense, Carex arctisibirica, Dryas octopetala, Festuca cryophila, Hedysarum arcticum \& Arctagrostis latifolia, sifting, 11.VIII.2014, leg. A.T.; $7 \sigma^{\top} \sigma^{\top}, 4$ $9+$, grass-moss meadow in valley with Polytrichum juniperinum, P. strictum, Pleurozium schreberi, Dryas octopetala, Equisetum arvense \& Festuca cryophila, sifting, 11.VIII.2014, leg. L.R.

REMARKS. Siberian-Nearctic arcto-alpine range. West of Yamal Peninsula known up to the delta of Pechora River [Mazura, 2000]. Biotopic preferences are not clear.

\section{Tmeticus nigriceps (Kulczyński, 1916)}

MATERIAL. Intra. EC9: $1 \sigma^{7}, 2$ ㅇ, sedge-grass-moss tundra with Salix glauca, S. phylicifolia, Eriophorum polystachion, E. media, Polygonum viviparum, Arctagrostis latifolia \& Carex arctisibirica, sifting, 18.VIII.2014, leg. L.R. EC16: $2 \sigma^{\top} \sigma^{7}, 2$ 20 , Salix glauca stands on wet terrace with $S$. nummularia, Peltigera sp., Carex arctisibirica \& Alopecurus alpinus, sifting, 13.VIII.2014, leg. L.R. \& A.T.; 1 \%, Salix glauca stands on gentle slope of river terrace with Carex arctisibirica, Arctagrostis latifolia \& Eriophorum media, sifting, 13.VIII.2014, leg. A.T.

REMARKS. Siberian arcto-alpine range. West of Yamal Peninsula known from Dolgy Ins., Pechora Sea only [Marusik, Eskov, 2009]. Biotopic preferences are not clear; probably, an intrazonal species.

\section{Walckenaeria korobeinikovi Esyunin et Efimik, 1996}

MATERIAL. Zonal. EC7: 1 , Ledum-sedge-undershrub tundra near Sabetta-Yakha River bank with Ledum decumbens, Cassiope tetragona, Carex arctisibirica, Vaccinium vitis-idaea \& Arctagrostis latifolia, sifting, 15.VIII.2014, leg. L.R. EC7: 1 +, sedgegrass-undershrub tundra near Sabetta-Yakha River bank with Cassiope tetragona, Calamagrostis holmii, Carex arctisibirica \& Deschampsia borealis, sifting, 15.VIII.2014, leg. L.R. 2 o+ , lichenmoss-undershrub polygonal tundra with Cassiope tetragona, Vaccinium vitis-idaea \& Carex arctisibirica, sifting \& pitfall traps, 23-25.VII.2013, leg. L.R. \& A.B.

Intra. EC4: 3 우, moss-grass-sedge ribbon-shaped associations in depressions among polygonal tundra with Arctagrostis latifolia, Carex arctisibirica \& Pleurozium schreberi, sifting, 1921.VII.2013, leg. L.R. \& A.B. EC9: $1 \sigma^{7}, 1$ \%, sedge-grass-moss tundra with Salix glauca, S. phylicifolia, Eriophorum polystachion, E. media, Polygonum viviparum, Arctagrostis latifolia \& Carex arctisibirica, sifting, 18.VIII.2014, leg. L.R. EC11: 1 , diverse herbs-sedge-lichen-moss tundra near lake with Salix glauca stands, Carex aquatilis, Aulacomnium turgidum \& Calliergon richardsonii, sifting, 11.VIII.2014, leg. A.T. EC16: $2 \sigma^{\top} \sigma^{\top}, 3$ 우, Salix glauca stands on wet terrace with $S$. nummularia, Peltigera sp., Carex arctisibirica \& Alopecurus alpinus, sifting, 13.VIII.2014, leg. L.R. \& A.T. EC17: 1 O', moss-grass-sedge ribbon-shaped associations in depressions among polygonal tundra with Pleurozium schreberi, Arctagrostis latifolia, Carex arctisibirica \& Poa alpina, sifting, 23-25.VII.2013, leg. L.R. \& A.B.; 1 + , swampy diverse herbs-sedge regularly inundated meadow near lake with Arctagrostis latifolia, Carex aquatilis, C. arctisibirica, Eriophorum polystachion, E. medium, Comarum palustre \& Ranunculus pallasii, in hummocks of Dicranum flexicaule \& Aulacomnium palustre, sifting, 24.VII.2013, leg. L.R. \& A.B. 
REMARKS. East Palaearctic, arcto-boreal range. A polytopic species inhabiting most landscape elements and plant communities.

Fam. Lycosidae (1)

\section{Alopecosa mutabilis (Kulczyński, 1908)}

MATERIAL. Zonal. EC2: 1,+ 3 juv., lichen-moss-undershrub small-polygonal tundra with Cassiope tetragona, Vaccinium vitis-idaea \& Equisetum arvense, sifting \& pitfall traps, 1719.VII.2013, leg. L.R. \& A.B.; $2 \bigcirc^{7} \sigma^{7}, 11$ 우, 3 subad., lichenmoss-undershrub polygonal tundra on gentle slope of a brook valley with Cassiope tetragona, Equisetum arvense, Arctagrostis latifolia \& Polytrichum sp., sifting \& pitfall traps, 17-19.VII.2013, leg. L.R. \& A.B.

Intra. EC4: 2 juv., lichen-moss-grass-undershrub tundra on river terrace with Cassiope tetragona, Equisetum arvense \& Arctagrostis latifolia, pitfall traps, 19-21.VII.2013, leg. L.R. \& A.B. EC5: 2 juv., wet sedge-grass-moss-undershrub tundra along a small river with Salix lanata, S. phylicifolia, Cassiope tetragona, Carex aquatilis \& C. rariflora, sifting, 19. VIII.2014, leg. L.R. EC6: 5 juv., swampy grass meadows in former riverbed depressions in floodplain of Sabetta-Yakha River with Eriophorum media, $\mathrm{Ca}$ lamagrostis holmii \& Carex aquatilis, sifting \& pitfall traps, 2123.VII.2013, leg. L.R. \& A.B. EC12: 1 O $^{7}$ subad., gentle slopes in depressions among diverse herbs-moss-lichen-undershrub tundra with Cassiope tetragona, Equisetum arvense, Carex arctisibirica, Dryas octopetala, Festuca cryophila, Hedysarum arcticum \& Arctagrostis latifolia, sifting, 11.VIII.2014, leg. A.T.; 2 juv., swampy diverse herbs-sedge regularly inundated meadow near lake with Arctagrostis latifolia, Carex aquatilis, C. arctisibirica, Eriophorum polystachion, E. medium, Comarum palustre \& Ranunculus pallasii, sifting \& pitfall traps, 23-25.VII.2013, leg. L.R. \& A.B.

Azonal. EC4: $1 \sigma^{7}$, sedge-grass sandy beach associations along a small river with Eriophorum media, Calamagrostis holmii, Carex rotundata \& Polytrichum sp., sifting \& pitfall traps, 1921.VII.2013, leg. L.R. \& A.B.

REMARKS. Siberian-Nearctic arctic range. An eurytopic species inhabiting all landscape elements and plant communities.

Fam. Tetragnathidae (1)

\section{Pachygnatha clercki Sundevall, 1823}

MATERIAL. Zonal. EC15: 1 juv., sedge-moss-lichen tundra on gentle slopes of hills with Salix nummularia, Carex arctisibirica \& Rubus chamaemorus, sifting \& pitfall traps, 17-27.VII.2013, leg. L.R. \& A.B.

Intra. EC6: $1 \sigma^{7}$, swampy grass meadows in former riverbed depressions in floodplain of Sabetta-Yakha River with Eriophorum media, Calamagrostis holmii \& Carex aquatilis, sifting \& pitfall traps, 21-23.VII.2013, leg. L.R. \& A.B. EC6: 1 O$^{7}, 1$, wet mosssedge tundra, pitfall traps, 12-15.IX.2013, leg. M.K. EC10: 2 오오, 1 juv., sedge-moss hummocky swamp with Carex aquatilis, $C$. rariflora, Comarum palustre, Eriophorum media, Aulacomnium turgidum, Calliergon richardsonii \& Mnium sp., sifting, 11.VIII.2014, leg. L.R. \& A.T. EC12: 2 +P, gentle slopes in depressions among diverse herbs-moss-lichen-undershrub tundra with Cassiope tetragona, Equisetum arvense, Carex arctisibirica, Dryas octopetala, Festuca cryophila, Hedysarum arcticum \& Arctagrostis latifolia, sifting, 11.VIII.2014, leg. A.T.; 1 + grass-moss meadow in valley with Polytrichum juniperinum, P. strictum, Pleurozium schreberi, Dryas octopetala, Equisetum arvense \& Festuca cryophila, sifting, 11.VIII.2014, leg. L.R. EC13: 1 juv., mosssedge-undershrub tundra with Rubus chamaemorus, Carex arctisibirica \& Calamagrostis holmii, sifting, 15.VIII.2014, leg. L.R. EC16: 2 우우 subad., Salix glauca stands on gentle slope of river terrace with Carex arctisibirica, Arctagrostis latifolia \& Eriophorum media, sifting, 13.VIII.2014, leg. A.T. EC17: 1 O$^{7}, 3$ ㅇ, 2 juv., swampy diverse herbs-sedge regularly inundated meadow near lake with Arctagrostis latifolia, Carex aquatilis, C. arctisibirica, Eriophorum polystachion, E. medium, Comarum palustre \& Ranunculus pallasii, sifting \& pitfall traps, 23-25.VII.2013, leg. L.R. \& A.B.

Azonal. EC5: 1 †, 1 juv., wet sedge-grass-moss-undershrub tundra along a small river with Salix lanata, S. phylicifolia, Cassiope tetragona, Carex aquatilis \& C. rariflora, sifting, 19. VIII.2014, leg. L.R. EC6: 1 \%, grass-sedge meadow on beach of SabettaYakha River with Calamagrostis holmii, Carex rotundata, sifting \& pitfall traps, 21-23.VII.2013, leg. L.R. \& A.B.

REMARKS. Holarctic polyzonal range. An eurytopic species inhabiting all landscape elements and plant communities.

\section{Fam. Thomisidae (1)}

\section{Xysticus albidus Grese, 1909}

MATERIAL. Zonal. EC2: $3 \sigma^{\top} \sigma^{7}$, lichen-moss-undershrub polygonal tundra on gentle slope of a brook valley with Cassiope tetragona, Equisetum arvense, Arctagrostis latifolia \& Polytrichum sp., sifting \& pitfall traps, 17-19.VII.2013, leg. L.R. \& A.B.

REMARKS. Palaearctic arctic range. A typical zonal species.

\section{Results and discussion}

Based on the extensive material from numerous localities and from almost all vegetation types, it is safe to conclude that the araneofauna of northern Yamal has been revealed rather adequately. In our opinion, 35 spider species found there constitute a quite representative number for the arctic tundra of a rather small territory with a smoothed terrain, making up not less than $80-90 \%$ of the local spider diversity. This figure is comparable, for example, with the well-studied araneofauna of Wrangel Island numbering 50 species [Khruleva, 2007; Marusik, Eskov, 2009]. The observed disparity ( 15 species) could apparently be attributed to the highly dissected topography of the island, which significantly increases a number of biotopes for spiders, and to the influence of a nearby Nearctic centre of species speciation.

Eight linyphiid species are registered for the spider fauna of Yamal Peninsula for the first time: Arcterigone pilifrons, Hilaira nivalis, Mecynargus tundricola, Mughiphantes sobrius, Paraglyphesis polaris, Perro polaris, Semljicola simplex and Silometopoides pampia.

It was hitherto argued that western Taimyr could be the westernmost distributional limit of Perro polaris and Semljicola simplex [Eskov, 1985; 1986]. The findings of these species in Yamal Peninsula allow us to significantly extend the known ranges of both species. A discovery of $S$. pampia in northern Yamal was completely unexpected. It was believed that this species was widespread in the arctic zone of the Nearctic Region, being restricted in the Palearctic Region to Chukotka only. West of Magadan Area, westward up to Yamal 
Table 1. Distribution of spiders across the zonal-landscape types of plant communities in northern Yamal Peninsula. Таблица 1. Зонально-ландшафтное распределение пауков на северном Ямале.

\begin{tabular}{|c|c|c|c|c|c|c|}
\hline \multirow{3}{*}{$\begin{array}{l}\text { Family, species } \\
\text { Семейства, виды }\end{array}$} & \multicolumn{6}{|c|}{$\begin{array}{c}\text { Plant communities } \\
\text { Растительные сообщества }\end{array}$} \\
\hline & \multirow{2}{*}{$\begin{array}{c}\text { Zonal } \\
\text { Зональные } \\
\text { Lichen-moss- } \\
\text { sedge- undershrub } \\
\text { tundra } \\
\text { Плакорная } \\
\text { тундра } \\
\end{array}$} & \multicolumn{4}{|c|}{$\begin{array}{c}\text { Intrazonal } \\
\text { Интразональные }\end{array}$} & \multirow{2}{*}{$\begin{array}{c}\text { Azonal } \\
\text { Азональные }\end{array}$} \\
\hline & & $\begin{array}{c}\text { Microdepressions } \\
\text { on watershed } \\
\text { Микропонижения } \\
\text { на плакоре }\end{array}$ & $\begin{array}{l}\text { Swamps } \\
\text { Болота }\end{array}$ & $\begin{array}{l}\text { Flood-plains } \\
\text { Поймы }\end{array}$ & $\begin{array}{l}\text { Meadows } \\
\text { Луга }\end{array}$ & \\
\hline \multicolumn{7}{|l|}{ Fam. Gnaphosidae } \\
\hline Micaria constricta & $30^{7} \sigma^{7}, 4$ 우우 & & $10^{2}, 1+9$ & & $10^{7}$ & \\
\hline \multicolumn{7}{|l|}{ Fam. Linyphiidae } \\
\hline Arcterigone pilifrons $*$ & $2+9$ & $3 \sigma^{7} \sigma^{7}, 6$ + & $9 \bigcirc^{7} \sigma^{7}, 8$ क & $30^{7} 0^{7}, 18$ 우 & $10^{7}, 1+$ & \\
\hline Bathyphantes humilis & & $2 \sigma^{7} \sigma^{7}$ & $10^{7}$ & $3 O^{7} O^{7}, 2$ 우 & $1+$ & $10^{7}$ \\
\hline Dactylopisthes video & & & & & & 1 운 \\
\hline Diplocephalus barbiger & $10^{2}, 1+$ & & $10^{x}, 1+$ & & $1+$ & $3 q$ \\
\hline Erigone arctica palaearctica & $\sim 80 \sigma^{7} \sigma^{\top} \&$ \& & $14 O^{3} O^{3}, 7$ OP & $9 \triangle^{x} O^{7}, 3$ O & $6 O^{7} O^{7}, 4$ + +9 & $5 O^{x} O^{x}, 2+9$ & $\sim 80 \mathrm{O}^{\top} \mathrm{O}^{\top} \&$ \&O \\
\hline E. psychrophila & $1+$ & & $2+9$ & $2 \sigma^{\top} \sigma^{\top}, 1+$ & & $\sim 60 \bigcirc^{\top} O^{\top} \&$ \& \\
\hline E. remota & & & & 1 㝏 & & \\
\hline E. tirolensis & & & & & $10^{7}, 4$ 우우 & \\
\hline Gibothorax tchernovi & $10^{7}$ & & $10^{7}$ & $60^{7} \sigma^{x}, 1+$ & $170^{7} \sigma^{7}$ & \\
\hline Halorates holmgreni & $30^{7} O^{7}, 3+9$ & $5 O^{7} O^{7}, 13++$ & 13 우우 & $35 \sigma^{7} \sigma^{7}, 48+9$ & $12 \sigma^{2} \sigma^{2}, 19+q$ & $\sim 70 O^{7} O^{7} \&$ \& \\
\hline H. spetsbergensis & $100^{7} \sigma^{7}$ & & & 1 우 & & \\
\hline Hilaira glacialis & 12 Or $^{7}, 10$ 우 & 15 O’ $^{7}, 20$ 우 & & & $4 \sigma^{7} \sigma^{7}, 16$ + & \\
\hline H. incondita & & $1 \bigcirc^{\top}, 2$ 우 & $17 \bigcirc^{7} \sigma^{7}, 4$ 우 & 5 Or $^{7}, 14$ 우 & 12 우 & $3 \sigma^{7} \sigma^{2}, 6$ 우 \\
\hline H. nivalis * & $3 \odot^{7} \sigma^{7}, 9$ + 9 & $60^{7} O^{7}, 14$ 우 & 6 우 & 10 O’ $^{7}, 22$ ○ᄋ & $3 \sigma^{x} \sigma^{x}, 1+$ & $10^{7}, 3$ + \\
\hline H. proletaria & & $2 O^{7} \sigma^{7}, 6+9$ & $2 O^{7} O^{7}, 5$ 우 & $10^{7}, 2$ 우 & & \\
\hline Hybauchenidium aquilonare & $1+$ & $2+9$ & & & & \\
\hline Masikia indistincta & $10^{7}, 3$ 우 & $20 \bigcirc^{7} \odot^{7}, 27$ 우 & 12 O $^{7}, 29$ 우 & $60^{7} O^{7}, 8$ 우 & $10^{7}, 10$ 우 & $2 \sigma^{x} \sigma^{x}, 1$ 우 \\
\hline Mecynargus tundricola $*$ & $3 \bigcirc^{7} \sigma^{\top}, 12$ ㅇ & $17 \bigcirc^{7} \sigma^{7}, 31$ 우 & $5 \odot^{7} \sigma^{7}, 1+$ & 2 우우 & $50^{7} O^{x}, 6$ 우 & \\
\hline Mughiphantes sobrius * & $10^{7}$ & & & & 2 우 & \\
\hline Oreoneta leviceps & & & $1 \overbrace{}^{\top}, 2$ + + & & & \\
\hline Paraglyphesis polaris $*$ & & & 1 운 & $10^{7}, 1$ ㅇ & & \\
\hline Pelecopsis parallela & & $2 O^{7} \sigma^{7}, 1+$ & $3+90$ & $3 \sigma^{7} \sigma^{7}, 1+$ & & \\
\hline Perro polaris $*$ & 5 우 & & & & 3 우우 & \\
\hline Semljicola alticola & $2 \sigma^{7} \sigma^{7}, 1$ 우 & $10^{x}, 1+$ & $10^{7}, 2$ 우 & $40^{7} 0^{7}, 53$ 우 & & $2+90$ \\
\hline S. arcticus & $4 \bigcirc^{7} \sigma^{7}, 4$ + +9 & $7 O^{7} O^{7}, 8$ क & $3 O^{x} \sigma^{7}, 2$ + & $4 O^{x} O^{x}$ & $20^{7} \sigma^{\top}, 4+9$ & \\
\hline S. barbiger & 3 우우 & $3 O^{7} \sigma^{7}, 8$ P & $10^{7}, 19$ 우 & $6 \sigma^{7} \sigma^{7}, 13$ 우 & & \\
\hline S. simplex * & $10^{7}, 25$ 우 & $2 O^{7} \sigma^{7}, 6$ + + & 4 우 & $2+90$ & 12 우우 & \\
\hline Silometopoides pampia * & & $10^{\top}, 3$ 우 & $1 \overbrace{}^{7}, 3$ 우 & $30^{7} \sigma^{7}, 6$ 우 & & \\
\hline Tarsiphantes latithorax & $10^{7}, 3$ 우 & & & & $8 \sigma^{7} \sigma^{7}, 10$ 우 & \\
\hline Tmeticus nigriceps & & $1 \bigcirc^{\top}, 2$ 우 & & $2 O^{7} \sigma^{7}, 3$ 우 & & \\
\hline Walckenaeria korobeinikovi & 4 우 & $2 \circ^{7} \sigma^{7}, 4$ + +9 & & $2 O^{7} \sigma^{7}, 5$ 우 & & \\
\hline \multicolumn{7}{|l|}{ Fam. Lycosidae } \\
\hline Alopecosa mutabilis & $\begin{array}{c}2 O^{7} O^{7}, 12 \text { + }{ }^{\prime}, 6 \\
\text { juv. }\end{array}$ & 1 juv. & 2 juv. & 4 juv. & $1 O^{x} O^{x}, 3$ juv. & $10^{7}$ \\
\hline \multicolumn{7}{|l|}{ Fam. Tetragnathidae } \\
\hline Pachygnatha clercki & 1 juv. & $1 \bigcirc^{7}, 1+, 1$ juv. & 2 우, 1 juv. & $2 \mathrm{v} 0^{2}, 3$ 우우 & 3 우 & 2 우 1 juv. \\
\hline
\end{tabular}


Table 1 (continued). Таблица 1 (продолжение).

\begin{tabular}{|r|c|c|c|c|c|c|}
\hline Fam. Thomisidae & & & & & & \\
\hline Xysticus albidus & $30^{\top} O^{\top}$ & & & & & \\
\hline TOTAL species: & 24 & 21 & 23 & 24 & 20 & 12 \\
\hline TOTAL samples: & 242 & 259 & 179 & 318 & 170 & 237 \\
\hline
\end{tabular}

* Звездочкой помечены виды, впервые найденные на Ямале.

* Species marked with an asterisk have been recorded from Yamal Peninsula for the first time.

Peninsula and southward to China and Mongolia, $S$. pampia was shown to be substituted by its congener $S$. sphagnicola [Eskov, Marusik, 1992; Tanasevitch, 2006]. Yet, the initial hypothesis that S. pampia inhabits the typical and arctic tundra, whereas the southern tundra and boreal biotopes are inhabited by $S$. sphagnicola has not been confirmed. A revision of the type and comparative materials (see above) has proved the identifications of almost all studied samples of both species to be correct. Thus, the entire zonal profile of Siberia, from the tundra in the north to the mountains of southern Siberia, is populated by S. sphagnicola. Contrary, as based on new findings, $S$. pampia shows a disjunctive distribution in the Palearctic Region: besides Chukotka the species also occurs in northern Yamal, almost being in contact with S. sphagnicola; the latter species was reliably recorded from the southern part of the Peninsula.

As for a taxonomic composition, almost $90 \%$ of the studied araneofauna are Linyphiidae, and of the 31 representatives of the family 29 belong to the Erigoninae; the only species of Linyphiinae belongs to Micronetinae.

In terms of regional composition, the spider fauna of northern Yamal is indeed Siberian, which sets it apart from the fauna of southern Yamal containing significantly more European elements.

In zonal terms, the spider fauna of northern Yamal has an arctic appearance and contains ten purely arctic species, such as Alopecosa mutabilis, Arcterigone pilifrons, Erigone arctica palaearctica, Gibothorax tchernovi, Halorates spetsbergensis, Hilaira nivalis, Mughiphantes sobrius, Semljicola arcticus, Silometopoides pampia and Xysticus albidus. Yet, 23 species have arcto-alpine or arcto-boreal distributional patterns. The remaining two species - Pachygnatha clercki and Pelecopsis parallela — are polyzonal, i.e. being distributed across most of the natural zones of the Palaearctic Region.

Table 1 shows the spider distribution between zonal-landscape types of plant communities in northern Yamal Peninsula. The majority of recorded species are poly- or eurytopic, i.e. the species inhabiting the entire landscape profile and all/most of the vegetation types, showing no preference to a specific landscape element or a plant community. There are only two typical zonal species, preferring the zonal vegetation types despite their most pessimal environmental conditions: viz., arctic Halorates spetsbergensis and Xysticus albidus.
Typical intrazonal species, i.e. those avoiding the extreme conditions and occurring in intrazonal communities with a smoother gradient of climatic factors, include the following five species: Bathyphantes humilis, Hilaira incondita, $H$. proletaria, Pelecopsis parallela and Silometopoides pampia.

For several species there are not enough data to identify their biotopic preferences: viz., Dactylopisthes video, Erigone remota, E. tirolensis, Hybauchenidium aquilonare, Oreoneta leviceps, Paraglyphesis polaris and Tmeticus nigriceps; yet they are likely to prefer intrazonal habitats.

Among the 10 purely arctic species, only two (as mention above) are typical zonal. One species - Silometopoides pampia - is typical intrazonal, and the remaining seven are poly- or eurytopic species.

Contrary to the original expectations with regards to species diversity, zonal tundra communities yield a comparable number of species to that of intrazonal biotopes and even surpass some of them. Of the 24 species inhabiting zonal plant communities, two species are purely arctic, but others are arcto-alpine or arcto-boreal. Intrazonal biotopes are inhabited by spiders of the whole spectrum of zonal-landscape preferences. Azonal communities are least diverse (12 species) due to the absence of moss and litter, i.e. the substrate required for the majority of tundra spiders.

ACKNOWLEDGEMENTS. We are sincerely grateful to Nadezhda Leonova (Moscow, Russia) for her kind help in the characteristics of vegetation, as well as to Alexander Bastrakov, Anatoly Babenko, Marina Khrisanova and Vladimir Onufriev (Moscow, Russia) whose collections have been used in the present study. Thanks also go to Dmitri Logunov (Manchester, UK) for editing the English of the final draft, as well as to Kirill Mikhailov (Moscow, Russia) for the opportunity to work with the collections of the ZMMU. This study was supported by the Russian Foundation for Basic Research, Project \#15-04-05964.

\section{References}

Chernov Yu.I. 1978. [The structure of the animal population of the Subarctic]. Moscow: Nauka Publ. 165 pp. [in Russian].

Dahl M. 1928. Spinnen (Araneae) von Nowaja Semlja // Rep. Sci. Res. Exp. Novaja Zemlya 1921. Oslo. No.36. S.1-39.

Eskov K.Yu. 1985. [Spiders of the tundra zone of the USSR] // V.I. Ovtsharenko (ed.). Fauna i ekologiya paukov SSSR. Trudy Zool. Inst. AN SSSR. Leningrad. Vol.139. P.121-128 [in Russian]. 
Eskov K.Yu. 1986. [On the spider genus Pero Tanasevitch, 1985 (Aranei, Linyphiidae)] // Zool. zh. Vol.65. No.11. P.17381742 [in Russian, with English summary].

Esyunin S.L., Efimik V.E. 1996. Catalogue of the spiders (Arachnida, Aranei) of the Urals. Moscow: KMK Sci. Press Ltd. 229 pp.

Holm A. 1973. On the spiders collected during the Swedish expedition to Novaya Zemlya and Yenisey in 1875 and 1876 // Zool. Scripta. Vol.2. No.3. P.71-110.

Khruleva O.A. 2007. [The peculiar fauna of terrestrial arthropods on Wrangel Island as a reflection of the specific environment] // Priroda ostrova Vrangelya: sovremennye issledovaniya. St. Petersburg: Asterion. P.136-181 [in Russian].

Marusik Yu.M., Eskov K.Yu. 2009. Spiders (Arachnida: Aranei) of the tundra zone of Russia // S.I. Golovatch, O.L. Makarova, A.B. Babenko, L.D. Penev (eds.). Species and communities in extreme environments. Festschrift towards $75^{\text {th }}$ anniversary and a laudatio in honour of Academician Yuri Ivanovich Chernov. Sofia-Moscow: Pensoft Publishers \& KMK Sci. Press. P.131-164.

Mazura N. 2000. 6.6. Spiders // Pechora Delta: Structure and Dynamics of the Pechora Delta Ecosystems (1995-1999). Syktyvkar, Ledystad. P.133-137.
Mazura N.S., Esyunin S.L. 2001. Fauna and biotopic distribution of the spiders (Arachnida: Aranei) in the tundra zone of the North-East of Russian Plain // Arthropoda Selecta. Vol.10. No.1. P.75-81.

Rebristaya O.B. 2013. [Flora of the Yamal Peninsula. Modern state and history of the formation]. St. Petersburg: LETI. 312 pp. [in Russian]

Tanasevitch A.V. 2006. Linyphiid spiders of the Norsky Nature Reserve, Amurskaya Area, Russian Far East (Arachnida: Aranei) // Arthropoda Selecta. Vol.14 (for 2005). No.4. P.347371 .

Tanasevitch A.V., Koponen S. 2007. Spiders (Aranei) of the southern tundra in the Russian Plain // Arthropoda Selecta. Vol.15 (for 2006). No.4. P.295-345.

Tanasevitch A.V., Koponen S., Kaarlejarvi E. 2009. Small collection of spiders (Aranei) from the Polar Urals and Yamal Peninsula, Russia // Arthropoda Selecta. Vol.17 (for 2008). No.3-4. P.185-190.

Responsible editor D.V. Logunov 